\title{
WestVirginiaUniversity
}

THE RESEARCH REPOSITORY @ WVU

Graduate Theses, Dissertations, and Problem Reports

2014

\section{Trunk Biomechanical Responses during Sudden Loading}

Jie Zhou

West Virginia University

Follow this and additional works at: https://researchrepository.wvu.edu/etd

\section{Recommended Citation}

Zhou, Jie, "Trunk Biomechanical Responses during Sudden Loading" (2014). Graduate Theses, Dissertations, and Problem Reports. 156.

https://researchrepository.wvu.edu/etd/156

This Thesis is protected by copyright and/or related rights. It has been brought to you by the The Research Repository @ WVU with permission from the rights-holder(s). You are free to use this Thesis in any way that is permitted by the copyright and related rights legislation that applies to your use. For other uses you must obtain permission from the rights-holder(s) directly, unless additional rights are indicated by a Creative Commons license in the record and/ or on the work itself. This Thesis has been accepted for inclusion in WVU Graduate Theses, Dissertations, and Problem Reports collection by an authorized administrator of The Research Repository @ WVU. For more information, please contact researchrepository@mail.wvu.edu. 


\title{
Trunk Biomechanical Responses during Sudden Loading
}

\author{
Jie Zhou
}

\begin{abstract}
Thesis submitted
to the Benjamin M. Statler College of Engineering and Mineral Resources at West Virginia University
\end{abstract}

in partial fulfilment of the requirements for the degree of

\author{
Master of Science in \\ Industrial Engineering \\ Xiaopeng Ning, Ph.D., Chair \\ Majid Jaridi, Ph.D. \\ Ashish Nimbarte, Ph.D.
}

Department of Industrial and Management Systems Engineering

Morgantown, West Virginia

2014

Keywords: Low Back Pain; Sudden Loading; Foot Placement; Loading Handling Position;

Uneven Ground Condition

Copyright 2014 Jie Zhou 


\section{ABSTRACT \\ Trunk Biomechanical Responses during Sudden Loading}

\section{Jie Zhou}

Back injury caused by sudden loading is a significant risk among workers who perform manual material handling tasks $(\mathrm{MMH})$. Therefore, it is critical to understand the effects of influential factors on back injury risks during sudden loading, and to develop load handling strategies that can reduce the biomechanical impacts to the spine caused by sudden loading. In this study we explored the effects of foot placement, load handling position as well as uneven ground conditions on trunk biomechanical responses under sudden loading event.

In the first experiment we investigated the effects of different foot placements and load asymmetry on trunk biomechanics during sudden loading. Fifteen subjects experienced sudden release of a $6.8 \mathrm{~kg}$ external load from symmetric or asymmetric directions while maintaining four different foot placements. The results showed that subjects experienced on average 4.1 degrees less trunk flexion, 6.6 Nm less L5/S1 joint moment and 32.0 N less shear force when using staggered stance with right foot forward (the most preferred placement) in comparison to wide stance (the least preferred placement). Asymmetric load releasing position consistently resulted in smaller trunk biomechanical impact than symmetric position. The findings suggest that staggered stance and asymmetric load holding position can be used as a protective load handling posture against low back pain caused by sudden loading.

In the second experiment we investigated the effects of load handling position on trunk biomechanics during sudden loading. Eleven male subjects were exposed to a $6.8 \mathrm{~kg}$ sudden loading while standing upright and holding the load at three different vertical heights in the sagittal plane or $45^{\circ}$ asymmetric to the sagittal plane. Results showed that subjects experienced smaller spinal compression with the decrease of load holding height; more specifically, at the 'Low (umbilicus level)' height condition, the peak L5/S1 joint compression force was $10.1 \%$ and $15.1 \%$ less than the 'Middle (shoulder level)' and 'High (eyebrow level)' conditions, respectively. Further, asymmetric posture resulted in $3.9 \%$ less compression force than symmetric posture. These findings suggest that handling loads in a lower position could work as a protective strategy when experiencing sudden loading.

In the third experiment we investigated the effects of uneven ground conditions on trunk biomechanical responses during sudden loading. Thirteen subjects experienced sudden loading with two different weights $(3.4 \mathrm{~kg}$ and $6.8 \mathrm{~kg})$ while standing on flat or laterally slanted ground conditions $\left(0^{\circ}, 15^{\circ}\right.$ and $\left.30^{\circ}\right)$. Our results showed that subjects experienced larger peak L5/S1 joint compression force with the increase of ground slanted angle. On average, the peak L5/S1 joint compression force generated in the $30^{\circ}$ condition was $6 \%$ and $8 \%$ larger than $15^{\circ}$ and $0^{\circ}$ conditions, respectively. Furthermore, greater trunk biomechanical impact was constantly observed in the $3.4 \mathrm{~kg}$ weight condition compared with the $6.8 \mathrm{~kg}$ condition. Findings of this study suggest that standing on laterally slanted ground surface increases the risk of low back injury when experiencing sudden loading. 


\section{TABLE OF CONTENTS}

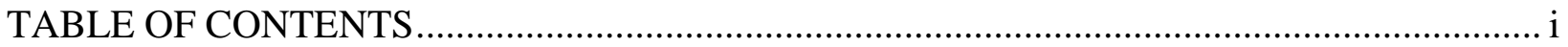

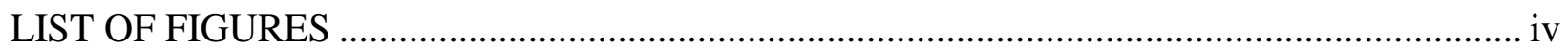

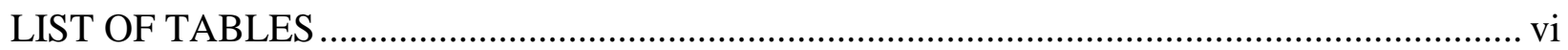

LIST OF ABBREVIATIONS ..................................................................................... vii

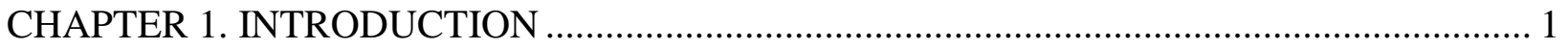

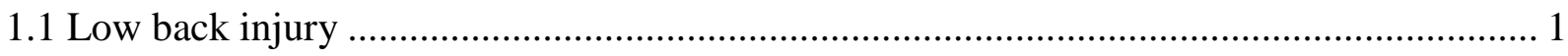

1.2 Low Back Pain Caused by Sudden loading .................................................................. 1

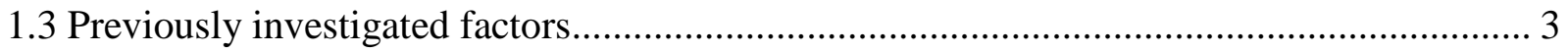

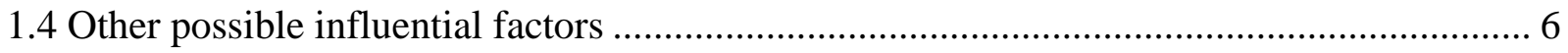

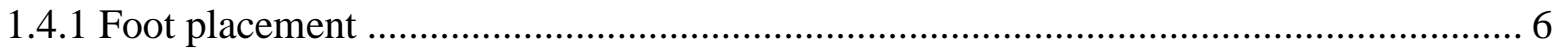

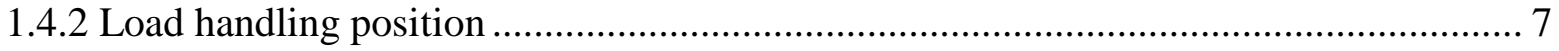

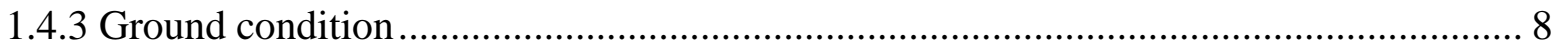

CHAPTER 2: RATIONALE AND OBJECTIVE ............................................................ 10

CHAPTER 3. STUDY OF THE EFFECT OF FOOT PLACEMENT …................................. 11

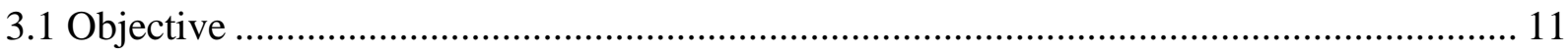

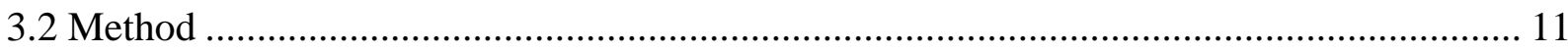

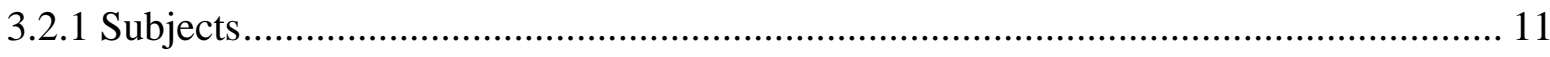

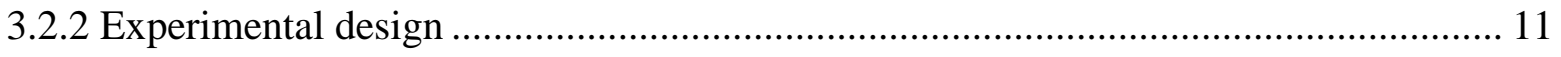

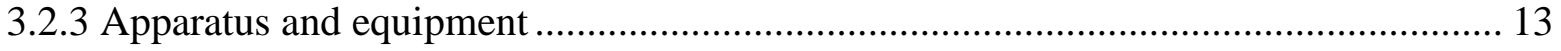

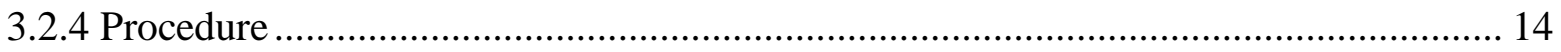




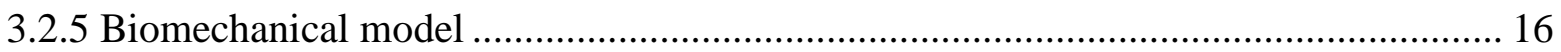

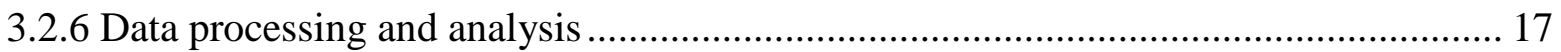

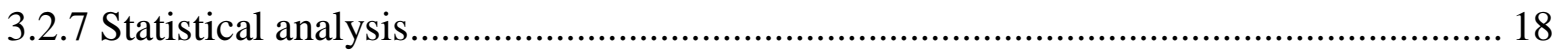

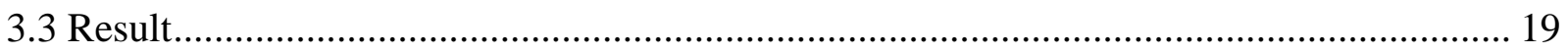

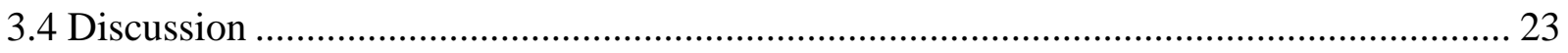

CHAPTER 4. STUDY OF THE EFFECT OF LOAD HANDLING POSITION ........................ 28

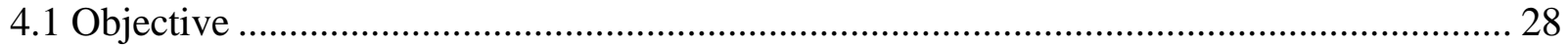

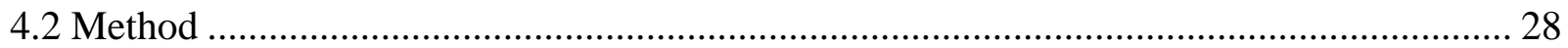

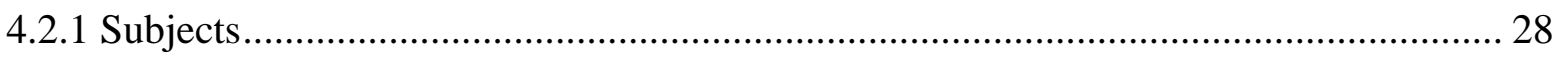

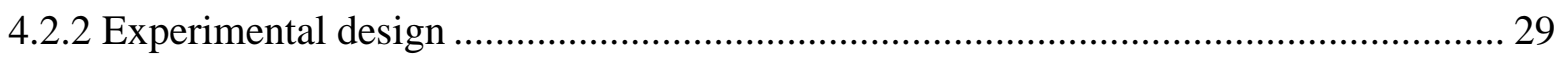

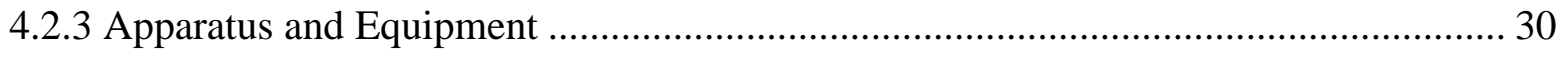

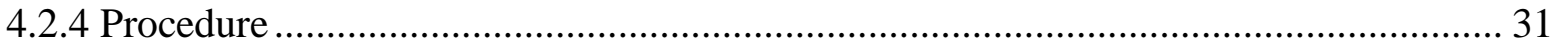

4.2.5 Data processing and analysis ................................................................................... 32

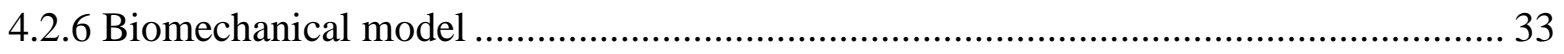

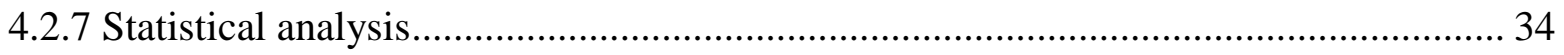

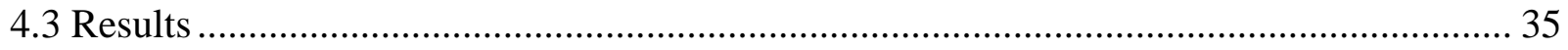

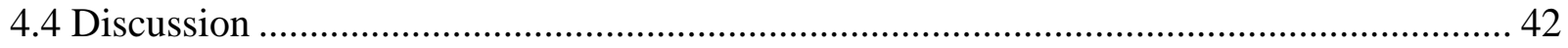

CHAPTER 5. THE STUDY OF THE EFFECT OF UNEVEN GROUND CONDITION ........... 46

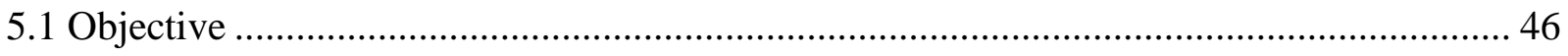

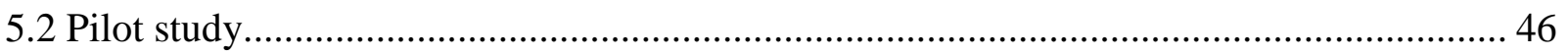

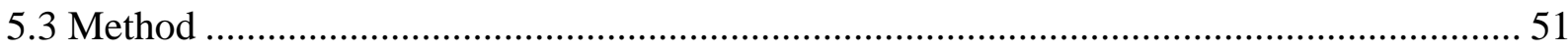

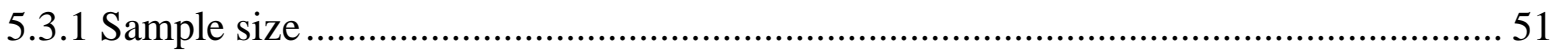




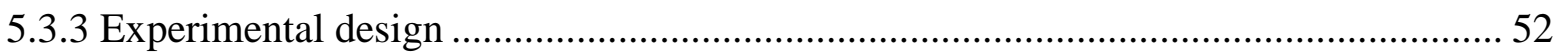

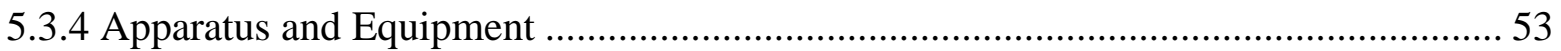

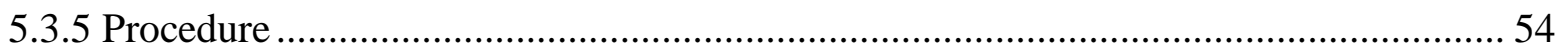

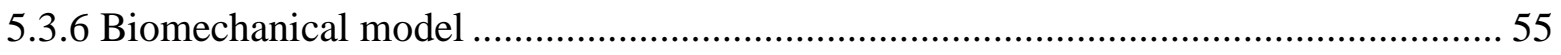

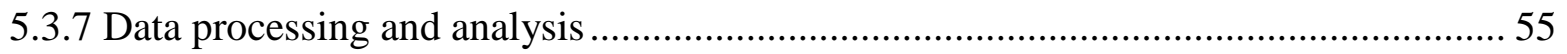

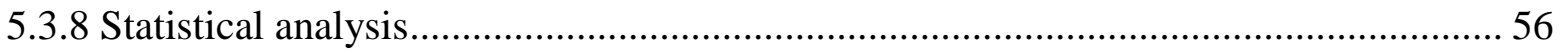

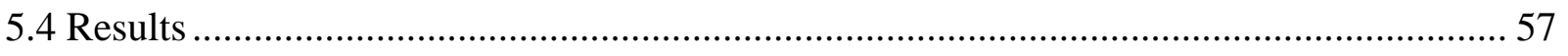

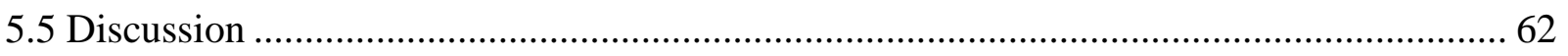

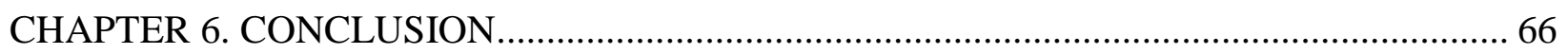

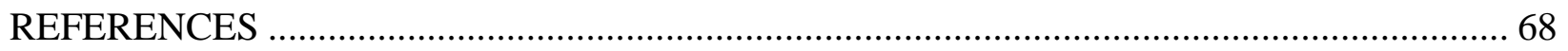

Appendix A: CONSENT AND INFORMATION FORM .............................................. 73 


\section{LIST OF FIGURES}

Figure 1. A diagram that demonstrates all eight experiment conditions. 13

Figure 2. (a) The hand load used in the experiment. (b) The placements of EMG electrodes and reflective markers. (c) A side view of the experiment setup (prior to the load releasing event).. 15 Figure 3. Peak increase in trunk flexion angle caused by sudden external loading under the four different foot placement conditions. 20

Figure 4. Peak increase in L5/S1 joint moment caused by sudden external loading under the four different foot placement conditions.

Figure 5. Peak L5/S1 joint shear force caused by sudden external loading under the four different foot placement conditions. 21

Figure 6. Peak L5/S1 joint compression force caused by sudden external loading under the four different foot placement conditions. Bars indicate the corresponding 95\% confidence interval. 22 Figure 7. A side view of the experiment setup (left panel) and a demonstration of different load handling positions (right panel). 30

Figure 8. Peak L5/S1 compression force caused by sudden loading under the three different load height levels. 37

Figure 9. Increase of trunk flexion angle caused by sudden loading under the three different load height levels. 37

Figure 10. Increase of L5/S1 joint moment caused by sudden loading under the three different load height levels. 38

Figure 11. Interaction between 'HEIGHT' and 'ASYM' for increase of trunk flexion angle. .... 39

Figure 12. Interaction between 'HEIGHT' and 'ASYM' for increase of L5/S1 joint moment.... 40 
Figure 13. Peak normalized EMG value (average from both sides) of trunk muscles caused by sudden loading under the three different load height levels (two ASYM levels combined)....... 42

Figure 14. Three different ANGLE conditions (A: 0 degree; B: 15 degree; C: 30 degree)........ 47

Figure 15. A side view of a sudden loading experimental trial: a subject is standing on slanted ground surface and holding the load before sudden loading event..................................... 49

Figure 16. Peak L5/S1 compression force under three different slanted angle conditions.......... 50

Figure 17. Peak L5/S1 compression force under three different weight conditions................... 50

Figure 18. Increase of trunk flexion angle caused by sudden loading under the three different

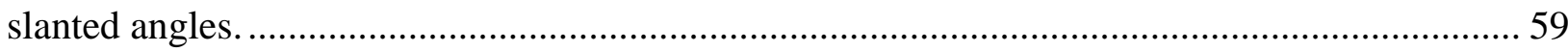

Figure 19. Increase of L5/S1 joint moment caused by sudden loading under the three different

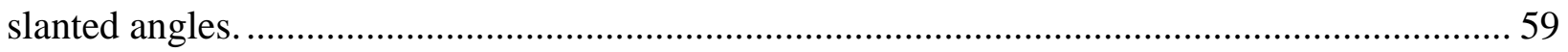

Figure 20. Peak L5/S1 compression force caused by sudden loading under the three different

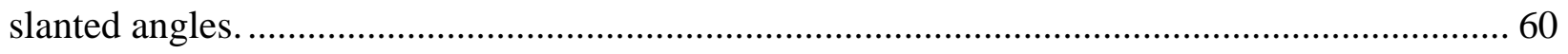
Figure 21. Averaged normalized EMG value (average of left and right sides with respect to MVC)

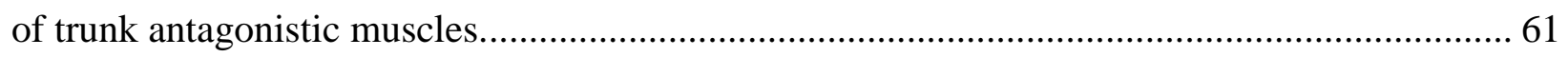
Figure 22. Normalized EMG pattern (with respect to MVC) of left and right sides of trunk agonist muscles 62 


\section{LIST OF TABLES}

Table 1. The results of MANOVA and univariate ANOVA................................................ 20

Table 2. The results of dependent variables at different ASYMMETRY conditions.................. 22

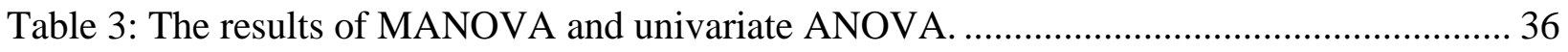

Table 4. The mean (SD) values of dependent variables at different ASYM conditions, p-values

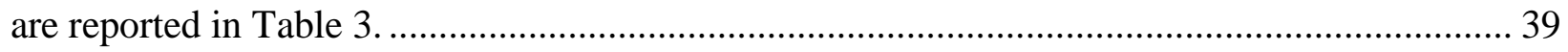

Table 5. The results of statistical analyses for related kinematics variables; mean (SD) values are

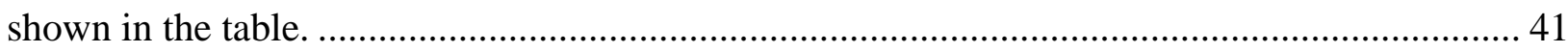

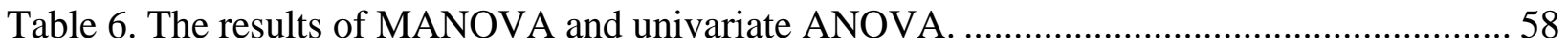

Table 7. The mean (SD) values of dependent variables at different WEIGHT conditions, $p$-values

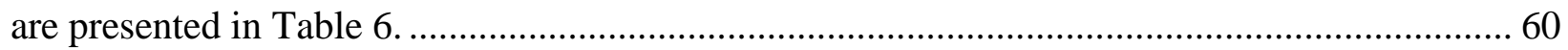




\section{LIST OF ABBREVIATIONS}

1. MMH. .manual material handling

2. LBP low back pain

3. EMG electromyography

4. IAP. intra-abdominal pressure

5. COP center of pressure

6. ML medial-lateral

7. AP anterior-posterior

8. FRP flexion relaxation phenomenon

9. SD standard deviation

10. PVC polyvinyl chloride

11. MVC .maximum voluntary contraction

12. ANOVA analysis of variance

13. MANOVA multivariate analysis of variance

14. ES .erector spinae

15. MU. multifidus

16. RA. rectus abdominis

17. EO .external oblique

18. NEMG normalized electromyography 


\section{CHAPTER 1. INTRODUCTION}

\subsection{Low back injury}

Work-related back injury is a worldwide health problem (Hoy et al. 2012). Each year in the United States, more than 10 million people will experience low back pain (LBP) (Marras 2000). According to national data, back injury accounts for $42 \%$ of all reported occupational musculoskeletal disorders with even higher rates reported for occupations that involve manual material handling tasks such as nurses (53\%), nursing aides (55\%), and labourers (44\%) (BLS 2013).

In addition to causing personal suffering, low back injury also resulted in significant financial cost. According to previous data, more than $30 \%$ of the total workers' compensation cost is related to low back injury (Webster and Snook 1994). Each year in the United States, work-related back injury causes over one hundred million lost work days (Guo et al. 1999) and billions of dollars of direct (e.g. medical treatment and workers' compensation) and indirect cost (e.g. lost productivity and the cost to hire temporary workers) (Frymoyer and Cats-Baril 1991; Maetzel and Li 2002; Stewart et al. 2003; Yelin and Callahan 1995).

\subsection{Low Back Pain Caused by Sudden loading}

During the performance of manual material handling tasks, sudden loading due to loss of control or external impact has been identified as a major contributing factor to back injury (Manning et al. 1984; Omino and Hayashi 1992). Manning and colleagues analyzed 1153 accident records that reported back injury in Ford Motor Company's Halewood Estate in 1980. Of all the 1153 back injuries, 401 workers had to require lost working days or restricted work; and $122(30.4 \%)$ of the 401 injuries involved sudden loading or loss of postural balance 
(Manning et al. 1984). Later, Omino and Hayashi investigated dynamic postures of airline attendants and its association with low back pain occurrence. From 98 low back pain reports, they identified six typical tasks that were closely related to LBP. It has been found that almost half of the cases involved sudden loading, including lifting light objects that looked apparently heavy, supporting unstable items, and sudden falling of objects. The author believed that appropriate preparatory strategy could prevent the risk of LBP (Omino and Hayashi 1992).

When human trunk experiences sudden impact, both reflexive and voluntary muscle contractions are initiated to increase stability and regain balance (Cholewicki et al. 1997). Reflexive contraction refers to an instinctive muscle response to external stimulus, and is initiated automatically without consciousness; on the other hand, voluntary contraction is purposely performed by a person, and is consciously initiated (Zedka et al. 1999). Such instantaneous muscle reaction elevates spinal loading, due to the fact that the spine, acting like a fulcrum, must withstand not only the external moment derived from gravity of the external load and body segments, but also the internal moment generated by muscle force to counterbalance the external load. And muscle force contributes a far greater portion of the total spinal loading compared with external load since muscles have a much shorter moment arm than an external load. (Granata and Marras 2000; Marras et al. 1987). As discussed below, such spinal loading is even larger when the load is unexpected (Marras et al. 1987) due to the overreaction of musculoskeletal system (Greenwood et al. 1976).

Previous studies have reported that the magnitude of spinal biomechanical loading (such as spinal compression and shear forces) is directly associated with the risk of back injury (Norman et al. 1998; Bakker et al. 2007). Norman and colleagues conducted a large scale casecontrol study on automotive workers, 130 randomly selected controls and 104 LBP cases were 
examined. Findings of this study showed that significantly higher spinal biomechanical loading was found in LBP cases than controls. And four most important risk factors were identified: peak shear force at the L4/L5 joint, peak trunk velocity, hand force and integrated lumbar moment (or compression) over a shift (Norman et al. 1998). Bakker et al (2007) also conducted a casecontrol study to evaluate daily spinal loading as a risk factor for acute LBP. The 24-Hour

Schedule (24 HS) questionnaire (developed to quantify spinal loading) was completed by 100 cases with acute LBP and 100 controls. It has been discovered that significantly higher $(p<0.0001) 24$ HS score was found in those who have acute LBP than control group, indicating that intensive spinal loading is strongly associated with acute LBP (Bakker et al. 2007).

\subsection{Previously investigated factors}

Several factors that affect people's trunk biomechanical responses during sudden external loading have been explored. It has been shown that expectation and the presence of waning signals prior to the sudden impact significantly reduced muscle activation level and spinal compression force (Marras et al. 1987; Lavender and Marras, 1995). Marras et al. (1987) explored the effect of expectation on trunk muscle response. Twelve subjects experienced sudden drop of weights $(5,10,15$, and $20 \mathrm{lb})$ under expected and unexpected conditions, while holding a box statically. Subjects were allowed to watch the load drop in expected condition, and were deprived of visual and auditory cues indicating when the weight was dropped. Muscle activities were collected from three pairs (left and right) of trunk muscles: latissimus dorsi, erector spinae and rectus abdominus. The muscle activities recorded in the corresponding static load holding task served as a baseline. It has been revealed that in expected condition, peak muscle activity across the six trunk muscles was 35\% larger than static load holding condition; 
while under unexpected condition, peak muscle activity was 1.2 to 3 times larger than expected condition ( $\mathrm{p}<0.05)$. In addition, longer duration of force exertion as well as more rapid increase in trunk force generation was observed in unexpected condition compared with expected condition. Later, Lavender and Marras (Lavender and Marras, 1995) examined the effect of warning signal on trunk muscle response. Four subjects held a canvas bucket and arrested a 53.4 $\mathrm{N}$ dropping weight with and without prior temporal warning. Force plate, Lumbar Motion Monitor and surface electromyography (EMG) were used to record biomechanical responses of subjects. Results of this study showed that with the presence of warning signal, by stiffening trunk muscle prior to sudden loading, three of the four subjects were able to decrease spinal compression force.

The effects of experience and training have also been investigated. Lavender and colleagues studies the influence of experience on trunk biomechanical responses during sudden loading (Lavender et al. 1993). Four subjects experienced 53.4 N dropping weight for thirty times while holding a canvas bucket. Each subject performed the thirty experimental trials five times on separate days. EMG data, force plate data, lumbar kinematics data and Intra-Abdominal Pressure (IAP) data were collected. It has been demonstrated that compared with the first experimental session, flexion angle and peak compression force decreased by $22 \%$ and $18 \%$, respectively in the final session. This result indicated that subjects were able to adopt better strategies to cope with sudden loading. Lawrence et al (2005) developed an adaptive system identification model to evaluate the effect of training during sudden loading tasks. Trunk kinematics and muscle EMG data collected from six subjects served as inputs of the model. In each sudden loading trial, subjects first maintained a $20^{\circ}$ trunk forward flexion posture, while they were able to keep trunk muscles relaxed since trunk weight was counterbalanced by an 
energized electromagnetic coupling. By de-energizing the electromagnetic coupling, an external impact caused by the subjects' trunk weight was suddenly applied. The results of this study demonstrated that after three days training, peak lumbosacral joint torque was significantly reduced by 25\% (Lawrence et al. 2005).

Fatigue is an important influential factor as well (Granata et al. 2004). Granata and colleagues developed a biomechanical model to evaluate the effect of fatigue on trunk muscle response and spinal stability. According to the model, fatigue resulted in reduced muscle stiffness, which necessitated increased co-contraction of trunk antagonistic muscles to maintain stability, and as a result of this co-contraction, increased spinal compression force was generated. In additional to the aforementioned theoretical modelling, empirical data (trunk muscle EMG and trunk kinematics data) were collected from twenty one subjects to examine the accuracy of the model. Subjects performed sudden loading tasks prior and after fatigue protocol, which required subjects to repeatedly lift a $12.7 \mathrm{~kg}$ load from the floor to an upright posture at a rate of 60 lifts/minutes for 2 minutes. In each sudden loading trial, subjects first maintained an upright standing posture against a $110 \mathrm{~N}$ horizontal preload (applied to their chest through harness), a $2.27 \mathrm{~kg}$ load was then suddenly dropped from 0.5 or $1.0 \mathrm{~m}$. Results of the empirical data supported model prediction, demonstrating significantly increased antagonistic muscle cocontraction and spinal compression force in fatigued conditions.

Stokes et al. (2000) investigated the effects of trunk muscle pre-activation level on muscle response during sudden loading (Stokes et al. 2000). Thirteen subjects maintained an upright standing posture with the pelvis restricted, preload and sudden load were provided through a chest harness. Subjects performed trunk muscle exertion of $20 \%$ or $40 \%$ of maximum voluntary extension contraction, while sudden loading was applied. EMG data from 12 trunk 
muscles were collected. It has been found that lower preload level (20\%) resulted in increased muscle response, and increasing spinal pre-activation could help stabilize the spine and reduce the risk of back injury during sudden external loading.

In summary, previous studies have demonstrated how expectation (warning signal), training, fatigue and trunk muscle pre-activation level could affect trunk biomechanics and the associated risk of low back pain during sudden loading. Findings of these studies could help develop strategies for handling sudden loading in an occupational setting, e.g. provide warning signal and training, avoid muscle fatigue. To mitigate the risk of back injury during manual material handling, it is critical to understand the effect of other influential variables, so as to develop load coping strategies that can reduce the biomechanical impacts to the spine caused by sudden loading.

\subsection{Other possible influential factors}

\subsubsection{Foot placement}

The influence of foot placements on stability has been investigated previously. Kirby and colleagues explored the association between foot placements and standing balance (Kirby et al. 1987). By using force plate, displacement of center of pressure (COP) when performing static standing tasks was examined from 10 subjects. Four variations of medial-lateral (ML) foot placements (feet together, feet 15, 30 and $45 \mathrm{~cm}$ apart), five variations of anterior-posterior (AP) foot placements (feet even, right foot ahead 10 and $30 \mathrm{~cm}$, left foot ahead 10 and $30 \mathrm{~cm}$ ), and five variations of foot angle (feet straight, toe-in 25 and $45^{\circ}$, toe-out 25 and $45^{\circ}$ ) were tested. In each trial, subject stood barefoot looking forward at a fixed point, and postural sway data of 20 second was collected. The results showed that the enlargement of foot placement in either 
medial-lateral or anterior-posterior direction increased displacement of COP in that direction; in addition, COP displacement in both ML and AP directions were smallest in the toe-out $25^{\circ}$ condition, and greatest in the tow-in $45^{\circ}$ condition.

\subsubsection{Load handling position}

Granata and Orishimo examined the influence of load holding height on trunk muscle activation level during static load hold task (Granata and Orishimo, 2001). A two-dimensional biomechanical model was developed to predict trunk muscle co-contraction when holding load at different height levels. The model showed that co-contraction of trunk antagonistic muscles must increase when holding load at a higher vertical level due to decreased stability. Empirical data (trunk muscle EMG and lumbar kinematics data) were collected from twenty subjects (ten male and ten female) to validate the model prediction. Subjects were asked to hold barbell of 4.5 or $9.0 \mathrm{~kg}$ at five different height levels $(0,20,40,60$ and $80 \mathrm{~cm}$ above L5/S1 joint), and two guidebars were used to assure that the horizontal moment arm of barbell was constant at $30 \mathrm{~cm}$ for all height conditions. Results of the experimental data supported model prediction, EMG activity of flexor muscle increased significantly $(\mathrm{P}<0.001$ and $\mathrm{p}<0.009$, respectively) with height of the load and magnitude of the load. Additionally, a significant $(\mathrm{p}<0.037)$ gender difference was observed, flexor muscle activity was $32 \%$ higher in female subjects than male subjects. The author pointed out that different load holding heights affected spinal stability, which changed trunk muscle activity.

In a follow up study, Granata and Wilson investigated the effect of trunk posture on spinal stability (Granata and Wilson, 2001). By building a three-dimensional model, and collecting empirical data from 10 subjects ( 5 male and 5 female), they demonstrated that spinal 
loading increased when holding load statically at an asymmetric trunk posture, compared with sagittally symmetric trunk posture. Additionally, a more flexed trunk posture helped increasing spinal stability, while it also resulted in larger spinal loading.

\subsubsection{Ground condition}

The effects of uneven ground condition on the trunk biomechanical responses have been investigated before. Jiang and colleagues investigated the effect of laterally slanted ground on trunk biomechanics during static load holding and dynamic lifting tasks (Jiang et al. 2005). Ten subjects performed static load holding task and dynamic load lifting task when standing on ground surfaces with lateral slant angle of $0^{\circ}, 10^{\circ}, 20^{\circ}$ and $30^{\circ}$. EMG data from three pairs (left and right) of trunk and low extremity muscles (longissimus, multifidus and vastus medialis) and kinematics data from five body segments (left and right arms, left and right legs and trunk) and external load were collected. Results of this study demonstrated that in static load holding task, trunk muscle activity increased with a larger slant angle, and substantial different EMG activation pattern was observed between contralateral (right) side and ipsilateral (left) side of muscles. In dynamic load lifting task, peak L5/S1 joint moment decreased with a larger slant angle, due to slower lifting motion.

Ning and Mirka explored the effect of ground surface motion on trunk biomechanics during lifting, lowering and static load holding tasks (Ning and Mirka 2010). Two levels of weight (5 and $10 \mathrm{~kg}$ ) and five ground moving conditions (specified by ground angular displacement and vertical acceleration) were tested to simulate ship motion. It has been shown that peak sagittal plane angular deceleration during lowering was significantly higher in moving conditions than in the stationary condition. Also, EMG activity of external oblique muscles was 
significantly higher in moving condition than static condition, which indicated an important role in stabilizing the trunk. In addition, the author suggested that even though the tasks were performed in sagittal plane, angular displacement of ground surface and vertical acceleration changed them to asymmetric tasks, therefore muscle activity patterns were found different between contralateral and ipsilateral sides.

A recent study examined the effect of laterally slanted ground on the onset of Flexion Relaxation Phenomenon (FRP) of trunk extensor muscles (Hu et al. 2013). Fourteen subjects performed sagittal trunk flexion/extension motion on three laterally slanted ground surfaces $\left(0^{\circ}\right.$, $15^{\circ}$ and $30^{\circ}$ ), while lumbar muscle activities and trunk kinematics were recorded. Results of this study demonstrated that with an increase in slant angle, flexion-relaxation phenomenon of trunk extensor muscles occurred up to $6.2^{\circ}$ earlier on ipsilateral (left) side, whereas the contralateral (right) side was not affected. Furthermore, the onset of muscle flexion-relaxation on ipsilateral side was earlier than contralateral side, and this angular difference increased with a larger slant angle. 


\section{CHAPTER 2: RATIONALE AND OBJECTIVE}

As discussed in the previous chapter, foot placement, load handling position and ground condition were found to significantly influenced people's biomechanical responses (e.g. kinetics and muscle activity) when performing other related biomechanical tasks (static standing, static load holding, dynamic load lifting etc.). It is believed that these factors would also significantly affect trunk biomechanical responses during sudden loading tasks through similar mechanisms (e.g. postural stability, muscle co-contraction).

As a result, the purpose of the current study is to investigate the effects of foot placement, load handling position and uneven ground surface on trunk biomechanical responses when experiencing sudden external loading, such that we can have a better idea about the influences of these factors and how to develop protective strategies to protect against back injury caused by the sudden loading. According to the findings of previous studies, it is hypothesized that:

1. The enlargement of foot placement would reduce the impact of sudden external loading and result in smaller trunk biomechanical responses.

2. One will experience greater spinal compression force, but smaller postural perturbation when a load is handled at a higher position and released suddenly.

3. The increase of slanted ground angle will increase trunk muscle activation level and thereby generate higher L5/S1 joint compression force when experience sudden drop of load. 


\section{CHAPTER 3. STUDY OF THE EFFECT OF FOOT PLACEMENT}

\subsection{Objective}

As described above, previous studies have demonstrated that foot placement has significant influence on stability (Holbein-Jenny et al. 2007, Kirby et al. 1987). Therefore, it is believed that foot placement also significantly affects trunk biomechanical responses during sudden external loading. The purpose of this experiment is to investigate the effect of foot placements on the trunk biomechanics when experiencing sudden loading, such that the most preferred foot placement that would reduce the risk of low back injury can be identified. According to the previous findings, we hypothesize that the enlargement of foot placement would reduce the impact of external loading and result in smaller trunk biomechanical responses due to the elevated standing stability.

\subsection{Method}

\subsubsection{Subjects}

Fifteen male subjects with average age, body height and body weight of 25.9 years (SD 1.75), $176 \mathrm{~cm}(\mathrm{SD} 4.4)$ and $70.9 \mathrm{~kg}$ (SD 6.9), respectively were recruited from the student population of West Virginia University. All subjects were in good physical condition without previous history of LBP or upper extremity injuries. Informed consent was obtained from subjects prior to their participation. The experiment procedure was approved by the office of research integrity and compliance of West Virginia University.

\subsubsection{Experimental design}

The design of experiment involved two independent variables: foot placements 
(POSTURE) and releasing position of the load (ASYMMETRY). Four different foot placements were tested; they were referred to as narrow stance (NS), wide stance (WS), staggered stance with left foot forward (SL) and staggered stance with right foot forward (SR). In the NS condition subjects stand with both feet together with $5 \mathrm{~cm}$ lateral clearance between the medial sides of their ankles. In the WS condition subjects were required to stand with $30 \mathrm{~cm}$ mediolateral clearance between the medial sides of their ankles. The SL and SR conditions required subjects to place left or right foot forward respectively with $30 \mathrm{~cm}$ medio-lateral and $40 \mathrm{~cm}$ anterior-posterior clearance between the medial sides of their ankles. A $6.8 \mathrm{~kg}(15 \mathrm{lbs})$ weight was used as the load in this experiment. The load was released from two different positions with respect to subjects: symmetry (with subject facing the load) and asymmetry 45 degree. The combination of the two independent variables created 8 conditions (demonstrated in Figure 1), and each condition was tested for 3 times (a total of 24 trials) in a completely randomized order. In all 8 testing conditions, the midpoint between the medial sides of ankles superimposed on the same location; the distance between this point and the projected center of mass of the external load was kept constant for all conditions. Therefore, a constant initial moment arm for external loading can be ensured (Waters et al. 1993).

The dependent variables were: 1 . Increase in trunk flexion angle, which was defined as the difference between the peak trunk flexion angle during the impact of sudden loading and initial trunk angle; 2. Increase in lumbosacral (referred to as L5/S1) joint moment, which was defined as the difference between the maximum L5/S1 joint moment during the impact of sudden loading and initial L5/S1 joint moment; 3. Peak L5/S1 joint compression force; 4. Peak L5/S1joint shear force. The peak compression and shear forces were defined as the maximum forces observed during sudden loading. 


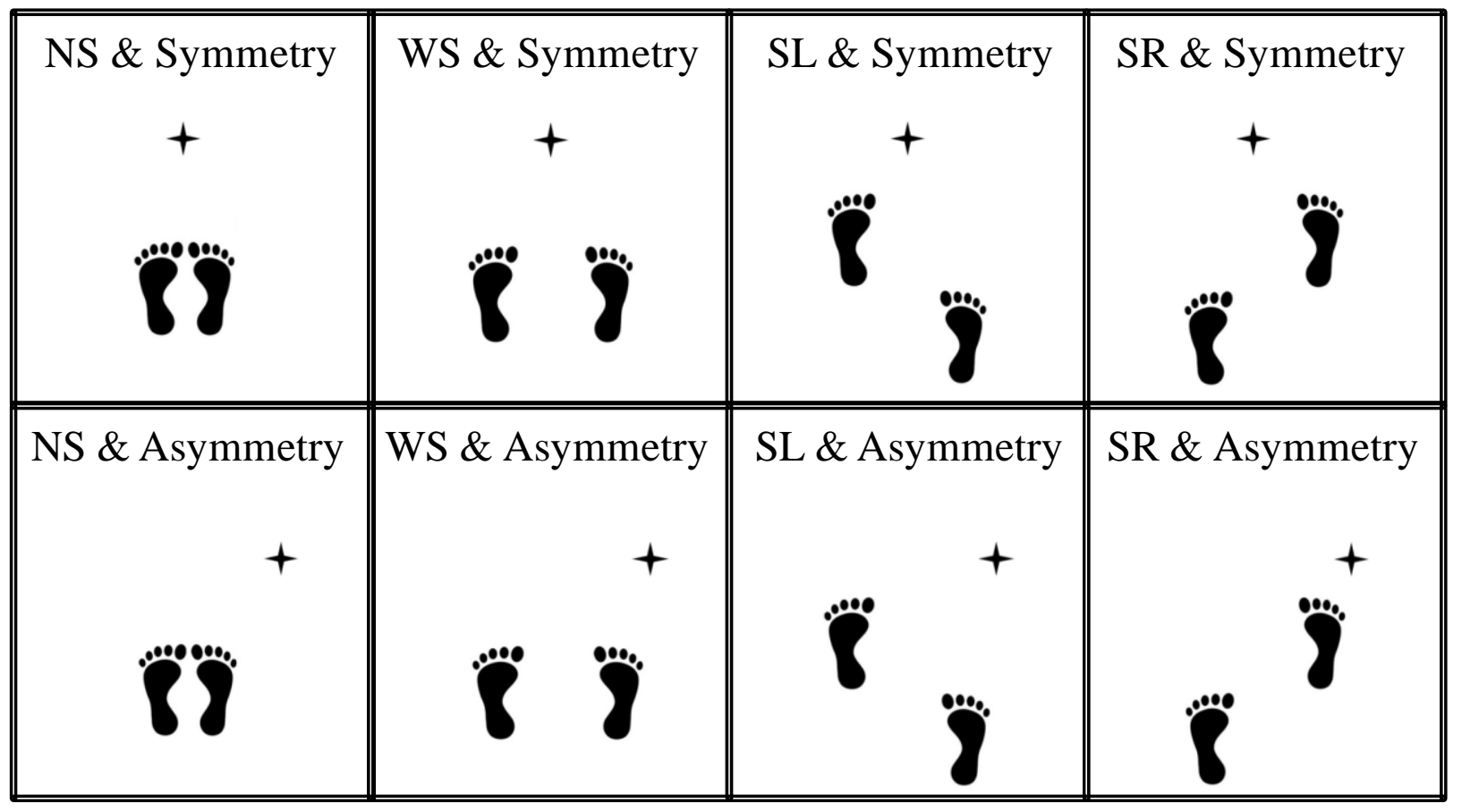

Figure 1. A diagram that demonstrates all eight experiment conditions.

\subsubsection{Apparatus and equipment}

A wood structure was created to hang the load at different height levels. The load itself was made from standard disc weights with a polyvinyl chloride (PVC) pipe used to secure the weights together through the central hole in the middle of the weights and to also serve as a handle for subjects to hold when performing the designated tasks (Figure 2 (a)).

A surface electromyography (EMG) system (Model: Bagnoli, Delsys Inc, Boston, MA, USA) was used to record EMG signals at $1000 \mathrm{~Hz}$ from eight trunk muscles: left and right rectus abdominis (electrodes placed $2 \mathrm{~cm}$ above the umbilicus and $3 \mathrm{~cm}$ to the midline of the abdomen); left and right external obliques $(10 \mathrm{~cm}$ to the midline of the abdomen and $4 \mathrm{~cm}$ above the ilium with an angle of $45^{\circ}$ to the midline of the abdomen); left and right erector spinae $(4 \mathrm{~cm}$ from the midline of L3 vertebra); left and right multifidus (2 $\mathrm{cm}$ from the midline of L4 vertebra). 
An eight camera (MX-13 series) 3D optical motion tracking system (Vicon, Nexus, Oxford, UK) was used to capture trunk and upper extremity kinematics at a sampling frequency of $100 \mathrm{~Hz}$. Seventeen reflective markers were placed over right and left shoulders: on the most dorsal points of acromioclavicular joints of both sides; along the spine: over C7, T12 and L5 vertebrae; right and left pelvises: over right and left sides of the anterior superior iliac spine; right and left elbows: on most caudal point on lateral epicondyle of both elbows; right and left hands: on the middle of the third metacarpal; right and left outsides of knees: on the lateral epicondyle of both knees; right and left outsides of ankles: on the lateral malleolus along an imaginary line that passes through the transmalleolar axis of both ankles; right and left toes: over the first metatarsal head, on the mid-foot side of the equinus break between forefoot and midfoot.

The placements of EMG electrodes and reflective markers are shown in Figure 2(b). Nexus 10.7 software (Vicon, Nexus, Oxford, UK) was used to record and synchronize both EMG and kinematics data.

During the trunk muscle maximum voluntary contraction (MVC) trials, a lumbar dynamometer (Humac Norm, CSMi, MA, USA) and the attached back flexion-extension module were used to restrict subjects' pelvis and lower extremity and provide static resistance against trunk muscle maximum exertions.

\subsubsection{Procedure}

Upon arrival of the subjects, experiment procedure was explained in detail and informed consents were obtained. Before the start of the experiment subjects' basic anthropometric data including body weight, height, trunk length, width and depth were measured. Next, a five minute 
warm-up routine was conducted to stretch and warm up trunk and upper extremity muscles. Subjects were then fitted with eight bi-polar EMG surface electrodes to the above mentioned sites and performed MVC trials in the back flexion-extension module. During the MVC, subjects were secured in a 20 degree trunk forward flexion posture and performed three repetitions of static trunk maximum extension/flexion exertions. Each MVC trial lasted five seconds and ample rest was given between trials in order to avoid muscle fatigue. The EMG activities of all sampled trunk muscles were recorded and used later as input to the biomechanical model (described in section 3.2.5).

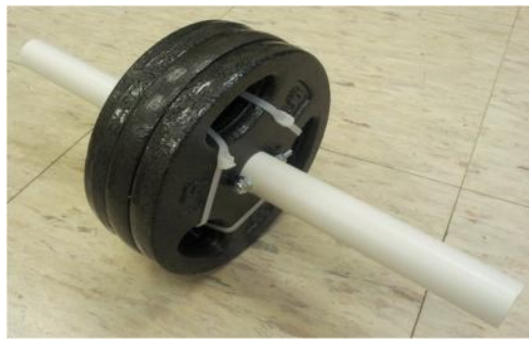

(a)

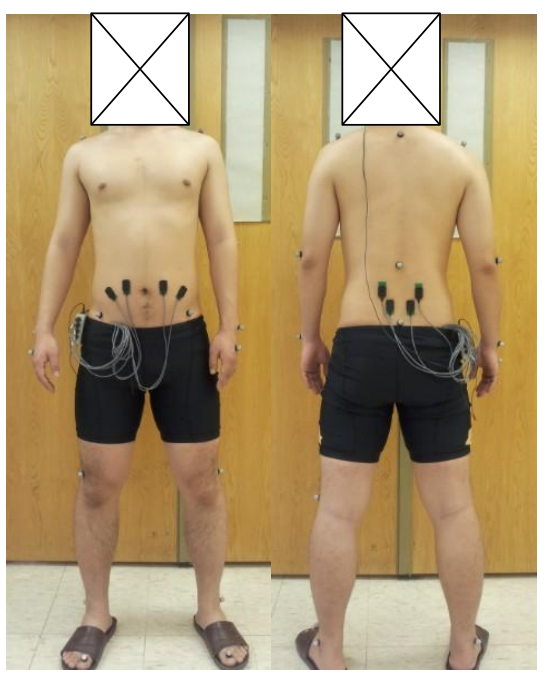

(b)

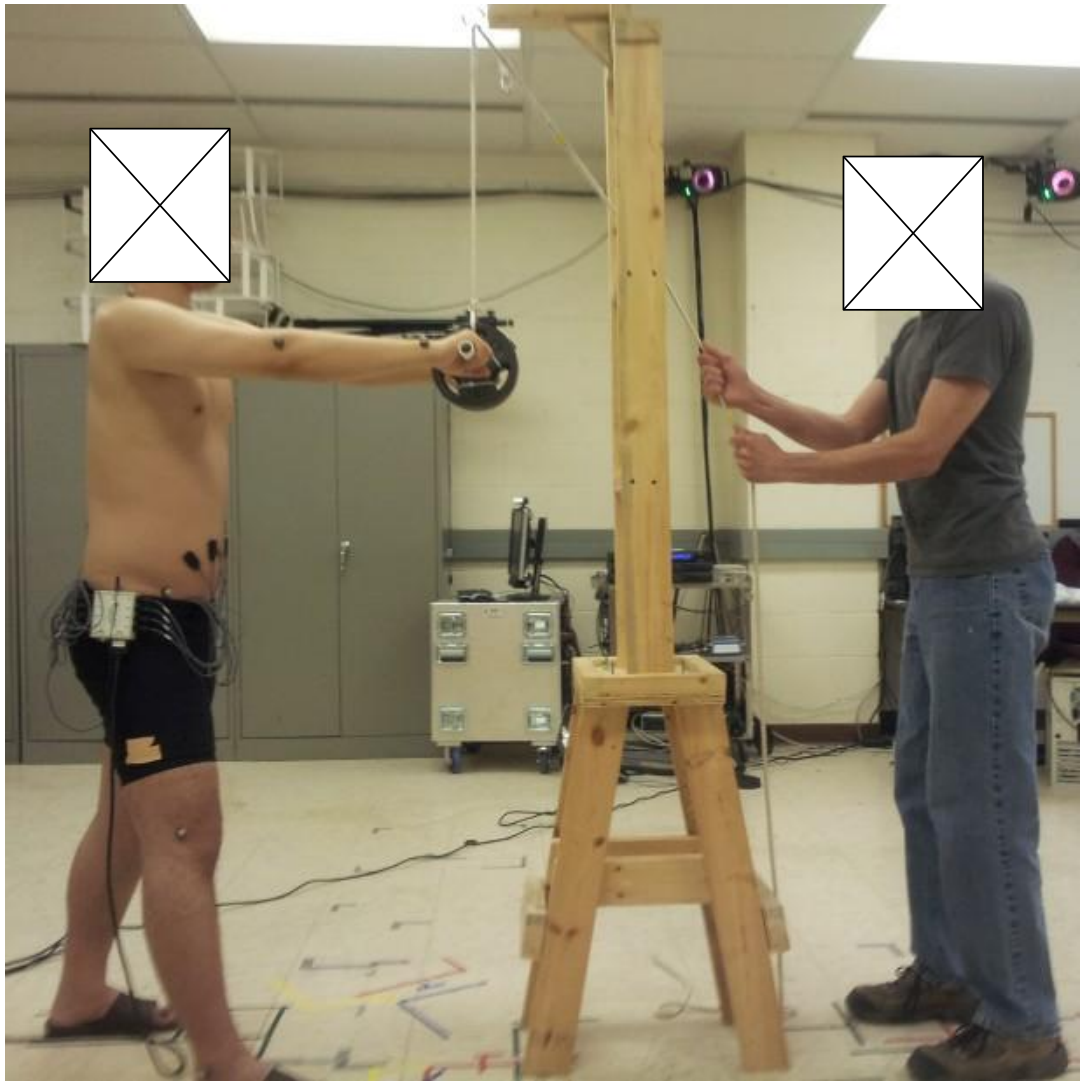

(c)

Figure 2. (a) The hand load used in the experiment. (b) The placements of EMG electrodes and reflective markers. (c) A side view of the experiment setup (prior to the load releasing event). 
After completing the MVC trials, nineteen reflective markers were secured to the designated locations and subjects performed all of the 24 experimental trials in a completely randomized order. In each trial, subjects first maintained the assigned foot placements, and then held the load steadily (without supporting the load) with both eyes closed. The experimenter who controlled the load would then release the load without notice (Figure 2(c)). Subjects were trained to respond to the sudden impact by carrying the load back to approximately their shoulder level (the original load height) as quickly as possible, and holding the load stably for three seconds. Ample rest was given between trials to avoid the accumulation of muscle fatigue.

\subsubsection{Biomechanical model}

The external moment about L5/S1 joint was estimated using a multi-segment dynamic motion model which consists of seven body segments (including trunk, upper arms, forearms and hands) and the load. Mass and the center of mass of each body segment were estimated according to previous studies (Drillis et al. 1964, Pheasant 1986). A previously established biomechanical model (Marras and Granata 1997) was used in the current study to estimate muscle forces, internal moment about L5/S1 joint and spinal compression and shear forces. In this model, the instantaneous tensile force generated by trunk muscle $i$ was estimated using equation (1) where "NEMG", " $G$ " and " $A$ " represent the normalized EMG (with regard to the MVC EMG), muscle gain and cross sectional area respectively; " $f\left(l_{i}\right)$ " and " $f\left(v_{i}\right)$ " are the muscle force-length and force-velocity modulation factors (Marras and Granata, 1997). The internal moment about L5/S1 joint was calculated using equation (2) where " $F$ " and " $r$ " are the force and moment arm vectors of muscle $i$ respectively. The L5/S1 joint compression and shear forces 
were then estimated based on trunk muscle forces, geometry (e.g. line of action) and trunk kinematics. Parameters such as moment arms and the cross sectional areas of all trunk muscles were estimated using regression equations summarized from early studies (Jorgensen et al. 2001; Marras et al. 2001). The muscle gain was obtained by matching the external and internal moment at the static load holding phase (the last three seconds) of all tasks. A best fit gain value was selected and applied to all muscles and trials for each subject.

$$
\begin{gathered}
F_{i}=N E M G_{i} \times G_{i} \times A_{i} \times f\left(l_{i}\right) \times f\left(v_{i}\right) \\
\vec{M}=\sum_{i}^{8} \vec{r}_{i} \times \vec{F}_{i}
\end{gathered}
$$

\subsubsection{Data processing and analysis}

EMG signals and reflective markers data were simultaneously recorded using Nexus 10.7 software. Trunk flexion angle was defined as the angle between the line connecting C7 and L5 marker and the transverse plane. External moment with respect to L5/S1 joint was calculated using the trunk and upper extremity kinematics data as well as anthropometric measurements as inputs to the multi-segment model. EMG data were filtered using $500 \mathrm{~Hz}$ low pass filter and $10 \mathrm{~Hz}$ high pass filter, signals were then notch filtered at $60 \mathrm{~Hz}$ (ambient electrical noise) and their aliases. After that the filtered EMG signals were rectified and smoothed with a 200 data points (0.2 second) sliding window. EMG signals from experimental trials were then normalized with respect to maximal EMG (collected from MVC trials) for each muscle. Finally normalized EMG profiles were used as inputs to the biomechanical model to estimate muscle forces, internal moment and spinal compression and shear forces. 


\subsubsection{Statistical analysis}

As demonstrated below, a general linear model was used to perform the statistical analysis.

$$
Y_{i j k}=\mu+\tau_{i}+\beta_{j}+\gamma_{k}+(\tau \beta)_{i j}+\varepsilon_{i j k l}
$$

Where $\mathrm{Y}_{\mathrm{ijk}}, \mu, \tau_{\mathrm{i}}, \beta_{\mathrm{j}}, \gamma_{\mathrm{k}}, \tau \beta_{\mathrm{ij}}$ and $\varepsilon_{\mathrm{ijkl}}$ represent biomechanical responses (dependent variables), overall mean, main effect of POSTURE, main effect of ASYMMETRY, block effect, interaction effect between POSTURE and ASYMMETRY, and random error. Total sum of squares was calculated as below:

$$
S S_{T}=\sum_{i=1}^{a} \sum_{j=1}^{b} \sum_{k=1}^{c} \sum_{l=1}^{n} Y_{i j k l}^{2}-\frac{y_{\ldots}^{2}}{a b c n}
$$

Sums of squares for the main effects and block effects were found as follows:

$$
\begin{aligned}
& S S A=\frac{1}{b c n} \sum_{i=1}^{a} y_{i . . .}^{2}-\frac{y_{\ldots .}^{2}}{a b c n} \\
& S S B=\frac{1}{a c n} \sum_{j=1}^{b} y_{. j . .}^{2}-\frac{y_{\ldots .}^{2}}{a b c n} \\
& S S C=\frac{1}{a b n} \sum_{k=1}^{c} y_{. . k .}^{2}-\frac{y_{\ldots .}^{2}}{a b c n}
\end{aligned}
$$


The interaction sum of squares was demonstrated as below:

$$
S S A B=\frac{1}{c n} \sum_{i=1}^{a} \sum_{j=1}^{b} y_{i j . .}^{2}-\frac{y_{\ldots . .}^{2}}{a b c n}-S S A-S S B
$$

The assumptions of the ANOVA model (normality of residuals, non-correlation of residuals, and constant variance of residuals) were first tested (Montgomery 2005). Dependent variables that violated one or more assumptions were transformed such that all assumptions were satisfied. Multivariate analyses of variance (MANOVAs) were then conducted on all variables of interest. Independent variables that were significant in the MANOVA were further analyzed in the univariate ANOVA. Tukey-Kramer post-hoc tests were performed on the main effect with more than two levels (i.e. POSTURE) to further investigate significance of difference between levels. The $\alpha$-value of 0.05 was used in all statistical tests as the level of significance.

\subsection{Result}

Result of MANOVA revealed significant main effect of both independent variables: POSTURE and ASYMMETRY; however their interactive effect was not significant therefore was not further tested. Univariate ANOVA demonstrated that both POSTURE and ASYMMETRY significantly affected the increase of trunk flexion angle, L5/S1 joint moment and the peak L5/S1 joint shear force (Table 1).

The results of post-hoc analysis revealed that the unexpected sudden external loading generated the largest increase of trunk flexion angle, increase of L5/S1 joint moment and shear force under WS condition, whereas the smallest impact was observed under the SR condition (Figure 3 to Figure 5, Different letters denote values that are statistically different from one 
another. Bars indicate the corresponding 95\% confidence interval). However the peak L5/S1 joint compression force was not affected by foot placements (Figure 6).

Table 1. The results of MANOVA and univariate ANOVA.

\begin{tabular}{|c|c|c|c|c|c|}
\hline \multirow[b]{3}{*}{ Independent Variables } & \multirow[b]{3}{*}{ MANOVA } & \multicolumn{4}{|l|}{ ANOVA } \\
\hline & & Trunk & $\mathrm{L} 5 / \mathrm{S} 1$ & $\mathrm{~L} 5 / \mathrm{S} 1$ & $\mathrm{~L} 5 / \mathrm{S} 1$ \\
\hline & & angle & moment & compression & shear \\
\hline POSTURE & $p<0.001$ & $p<0.001$ & $p<0.001$ & $p=0.41$ & $p<0.001$ \\
\hline ASYMMETRY & $p=0.039$ & $p<0.001$ & $p=0.002$ & $p=0.85$ & $p<0.001$ \\
\hline POSTURE*ASYMMETRY & $p=0.65$ & N/A & N/A & N/A & N/A \\
\hline
\end{tabular}

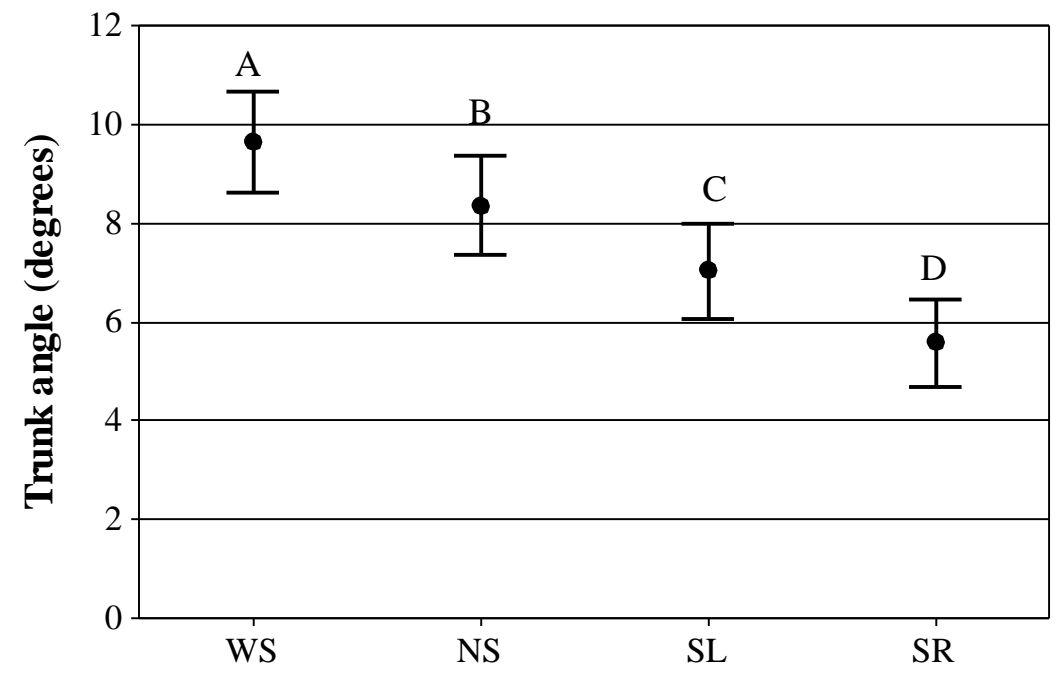

Figure 3. Peak increase in trunk flexion angle caused by sudden external loading under the four different foot placement conditions. 


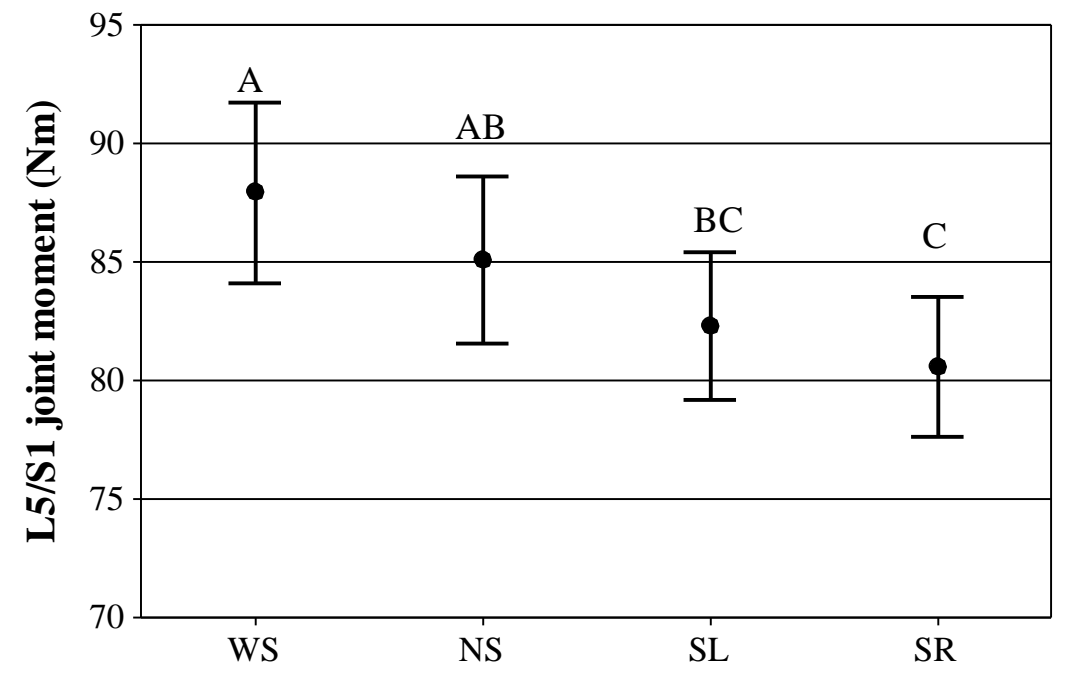

Figure 4. Peak increase in L5/S1 joint moment caused by sudden external loading under the four different foot placement conditions.

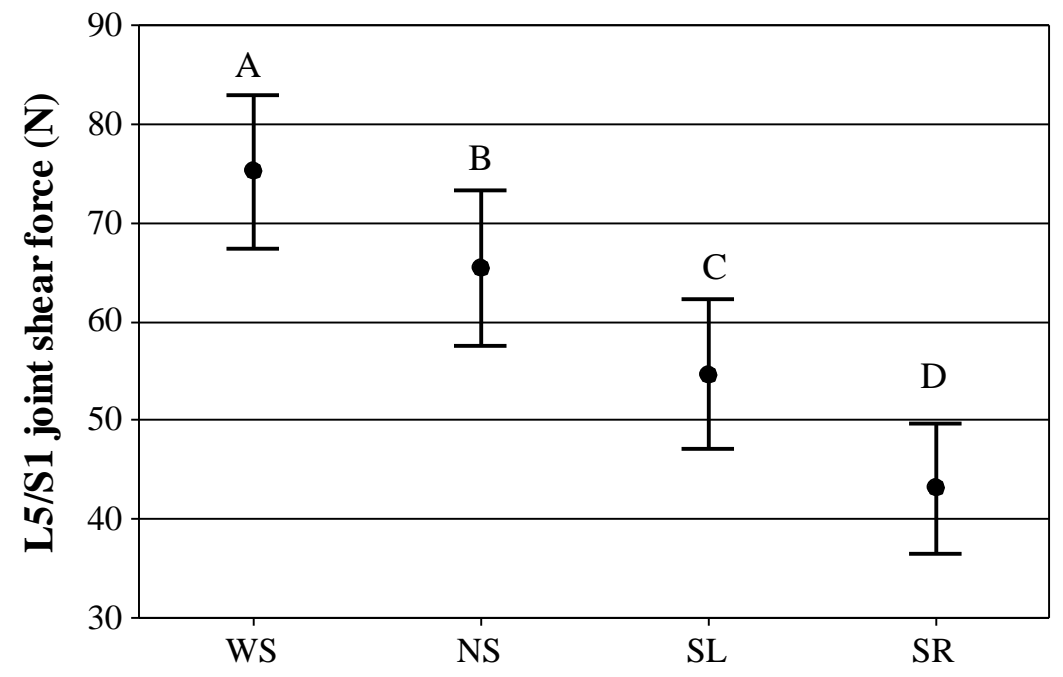

Figure 5. Peak L5/S1 joint shear force caused by sudden external loading under the four different foot placement conditions. 


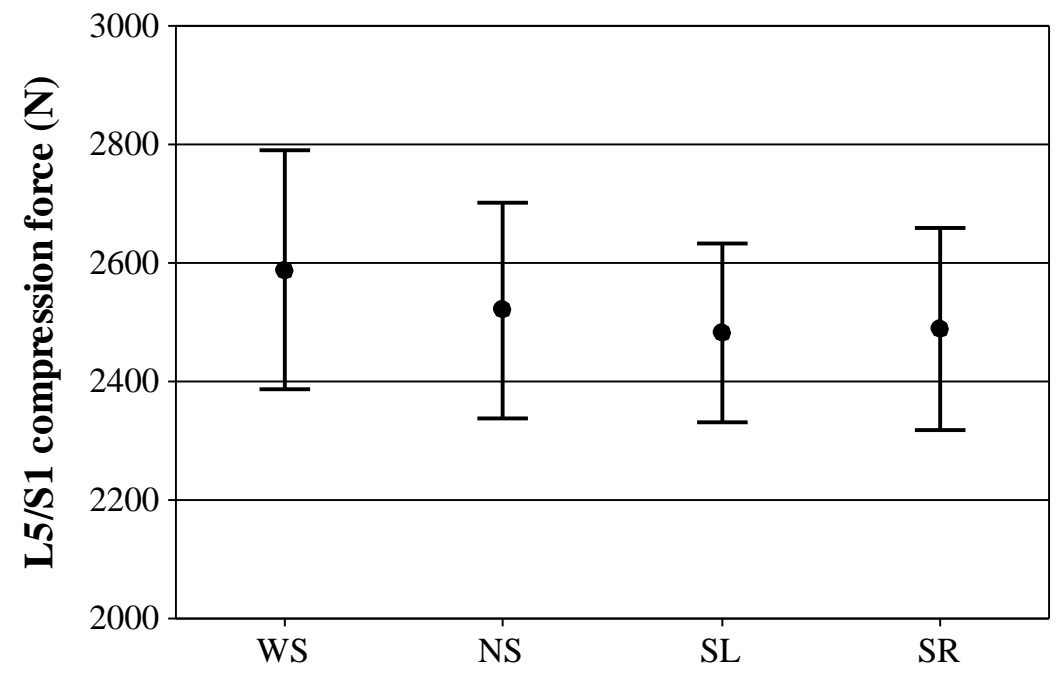

Figure 6. Peak L5/S1 joint compression force caused by sudden external loading under the four different foot placement conditions. Bars indicate the corresponding $95 \%$ confidence interval.

When comparing between the symmetric ( 0 degree) and asymmetric (45 degree) conditions, smaller increases in trunk flexion angle, L5/S1 joint moment and shear force were constantly observed in the asymmetric condition (Table 2). However the peak L5/S1 joint compression force was not affected by ASYMMETRY either.

Table 2. The results of dependent variables at different ASYMMETRY conditions

\begin{tabular}{lllll}
\hline ASYMMETRY & $\begin{array}{l}\text { Trunk angle } \\
(\text { degrees })\end{array}$ & $\begin{array}{l}\text { L5/S1 moment } \\
(\mathrm{Nm})\end{array}$ & $\begin{array}{l}\text { L5/S1 compression } \\
(\mathrm{N})\end{array}$ & $\begin{array}{l}\text { L5/S1 shear } \\
(\mathrm{N})\end{array}$ \\
\hline Symmetry & $\mathbf{8 . 4}$ & $\mathbf{8 5 . 5}$ & 2515.2 & $\mathbf{6 5 . 4}$ \\
\hline Asymmetry & $\mathbf{7 . 0}$ & $\mathbf{8 2 . 6}$ & 2525.1 & $\mathbf{5 2 . 3}$ \\
\hline
\end{tabular}

Notes: Bolded numbers indicate that results are significantly different between symmetry and asymmetry conditions, $p$-values are reported in Table 1. 
After having the results, a power test was performed to see if the sample size is enough. Operating characteristics curves (OC curve) were used to determine the power of the test. The parameter $\Phi$ was calculated using the below equation.

$$
\Phi^{2}=\frac{b n \sum_{i=1}^{a} \tau_{i}^{2}}{a \sigma^{2}}
$$

Where $\mathrm{n}, \mathrm{a}, \mathrm{b}, \tau$, and $\sigma$ represents the number of observations, levels of treatment a, level of treatment $\mathrm{b}$, the difference between treatment $i$ and treatment mean, and standard deviation in responses, respectively. It has been shown that the smallest power of test was 0.60 , when investigating the main effect of ASYMMETRY on peak L5/S1 joint compression force. According to this result, a larger sample size is necessary to obtain a design having a high probability of detecting the difference in peak L5/S1 joint compression force between two different ASYMMETRY conditions.

\subsection{Discussion}

Results of this study showed that foot placement had significant impact on trunk biomechanics during unexpected sudden loading which confirmed our initial hypothesis. Overall, the SR was the most desired foot placement, as it posted the smallest increase in trunk flexion angle, L5/S1 joint moment and peak L5/S1 joint shear force during sudden loading. The SL placement was the second most preferable placement followed by NS and WS which posted the largest trunk flexion and spinal loading. These results were likely generated by different lower extremity structures associated with different foot placements. In the two staggered foot placements, subjects placed one foot forward and one foot backward to form a geometrically 
stable triangular structure along the line of external load and L5/S1 joint. The ground reaction force (perpendicular up from the ground) acting on the frontal foot provided additional moment (about L5/S1 joint) that could help counterbalance the rotational moment created by the external load. Under the SR asymmetric load releasing condition subject's frontal foot has a larger moment arm (along the direction from L5/S1 joint to the external load) than any other conditions. This mechanism could explain why subjects experienced the smallest increase in trunk flexion, L5/S1 joint moment and shear force under this condition.

From the stability point of view, Kirby and his colleagues demonstrated that with the increase of feet separation distance in either anterior-posterior or medial-lateral direction, the envelope of COP sway also increased along that direction (Kirby et al. 1987). According to a recent study, the increased COP envelop caused by larger foot separation could indicate elevated postural stability (Holbein-Jenny et al. 2007). In the current study, the two staggered foot placements had the largest foot displacement which may result in the highest level of standing stability among all four foot placements. This could explain the smaller trunk biomechanical responses observed under SL and SR conditions.

Based on the results of this study, it was interesting to find that unexpected sudden loading generated lower trunk biomechanical impact when participant maintained NS than WS foot placement. Wide foot stance is commonly considered as a more stable and preferred placement when performing manual material handling tasks (Cholewicki et al. 1991, Sorensen et al. 2011). Like staggered foot placement, WS also creates a triangle between both legs and the ground, however, this triangular structure in the WS posture is medio-laterally oriented, therefore provides little resistance to the external loading along the anterior-posterior direction. Furthermore, previous studies have discovered significant forward shift of COP when subjects 
maintain wide foot stance compare with narrow stance (Kirby et al. 1987). This forward movement of COP may have resulted from the forward leaning of trunk which could potentially increase the magnitude of trunk flexion when subjects experience unexpected sudden impact in the anterior direction. It was also suspected that the increase of stance width may alter hip joints stiffness, which in turn could influence trunk biomechanical responses during sudden loading. However, such effect has not been investigated previously. Therefore, it warrants future research efforts.

Regarding load releasing position, our results demonstrated that asymmetric position generated significantly less increase in trunk flexion angle, L5/S1 joint moment and smaller peak L5/S1 joint shear force compared with symmetric position. Under symmetric load condition the direction of external moment is perpendicular to the rotating axis of pelvis (imaginary line that connects two hip joints) whereas, in asymmetric condition a $45^{\circ}$ angle was formed; it means that the moment arm between external load and the axis of pelvis rotation is larger in symmetric conditions. Therefore, the same external loading would generate larger pelvis rotational moment and angular displacement and consequently, larger increase of L5/S1 joint moment and peak L5/S1 shear force, under symmetric load conditions.

Although most dependent variables were significantly affected by both independent variables, the peak L5/S1 compression was an exception. Results of this study showed that neither POSTURE nor ASYMMETRY significantly affected the peak L5/S1 compression force due to the high variance observed in the estimated spinal compression force. But, we can still observe that, in general, WS and NS foot placements generated some higher peak L5/S1 compression force than the two staggered foot placements. These results are in line with the results of other dependent variables. Spinal compression is affected by both trunk kinematics and 
trunk muscle co-contraction (Granata and Wilson, 2001). Given a constant level of external moment, infinite number of possible agonist and antagonist trunk muscle contraction combinations could exist. In such, for a given trunk posture and external moment, spinal compression force could change significantly. Previous studies have suggested that, trunk muscle co-contraction elevated with decreased perceived stability (Granata and Wilson, 2001). In the current study, it is possible that variance in subjects' perceived stability changed their trunk muscle co-contraction levels which resulted in higher variance in spinal compression.

The effect of ASYMMETRY on spinal compression was also found to be insignificant despite the fact that clear differences were observed among other dependent variables (Table 2). According to trunk biomechanical model (Marras and Granata, 1997) the increase in trunk flexion angle elevates the magnitude of external moment about L5/S1 joint therefore should increase back extensor muscle force exertion. Based on this assertion, higher compression force should be observed in the symmetric load condition. However, another study also reported that subjects maintained higher trunk muscle co-contraction when holding load in asymmetric posture (Granata and Wilson. 2001). It is our belief that in the current study, the effect of larger trunk flexion in symmetric condition and higher muscle co-contraction in asymmetric condition counterbalanced each other and resulted in similar levels of trunk muscle activity, and consequently, similar spinal compression force.

Several limitations of the current study should be noted. First, the expectation of the load releasing event was controlled by having subjects close their eyes and keep muscles "relaxed" before the load drops. However, during the experiment, subjects may still try to anticipate the weight dropping thereby unintentionally elevate trunk muscle co-contraction. This could also explain the higher variance in spinal compression force that we have observed. Second, a 
relatively light (i.e. $6.8 \mathrm{~kg}$ ) load was selected for testing in order to avoid the accumulation of lumbar muscle fatigue and reduce the risk of injury. Due to the size of the load, trunk biomechanical differences between experimental conditions were often statistically significant but relatively small. In real occupational settings, much heavier loads may be experienced and larger differences could be revealed. Third, $45^{\circ}$ was the only asymmetry condition tested in the current study; other asymmetric angles were not studied. Finally, relatively small sample size (15 subjects) was used in the current study, future studies may use larger sample sizes to reveal the differences in spinal compression force between different foot placement conditions. 


\section{CHAPTER 4. STUDY OF THE EFFECT OF LOAD HANDLING POSITION}

\subsection{Objective}

The second study was designed to understand whether load handling position can be used in the design of a protective strategy against back injury caused by sudden loading. More specifically, the objective of the second experiment is to investigate the effect of load handling height and load asymmetry (created by arm rotation but not trunk rotation) on trunk biomechanics when experiencing sudden external loading. Based on the findings of previous studies, it is hypothesized that one will experience greater spinal compression force, but smaller postural perturbation when a load is handled at a higher position and released suddenly. Also as suggested by previous research (Zhou et al. 2013 ${ }^{\mathrm{a}}$ ), we expect to observe smaller trunk flexion when the load is handled and suddenly released from an asymmetrical position than in a symmetrical position.

\subsection{Method}

\subsubsection{Subjects}

Eleven male subjects with mean (SD) height, weight, and age of 176.6 (3.4) cm, 71.2 (6.5) $\mathrm{kg}$, and 26.7 (2.0) years, respectively, volunteered to participate in this study. Subjects with no previous training or working experience in manual material handling were recruited from the student population of West Virginia University, and none reported a previous history of low back pain or upper/lower limb injuries. The experimental procedure was approved by the university's Research Integrity and Compliance Committee. 


\subsubsection{Experimental design}

Two independent variables were involved in the current experimental design: vertical load handling position (HEIGHT, three levels) and transverse load handling position (ASYM, two levels). These two independent variables collectively describe the location of both hands and the load (the load was always in the hands (Figure 7) before the sudden releasing event). In all testing conditions, trunk remained forward facing. The three HEIGHT levels were defined with respect to participants' anthropometry: 'High' (eyebrow level: the initial holding height of the load aligns with the height of eyebrows), 'Middle' (shoulder level: the initial holding height of the load aligns with the height of clavicle bone), and 'Low' (umbilicus level: the initial holding height of the load aligns with the height of umbilicus). The two ASYM load positions were: 'Symmetry' (in the midsagittal plane) and 'Asymmetry' ( $45^{\circ}$ leftward to the midsagittal plane). The effect of transverse load handling location has been previously investigated (Zhou et al. $2013^{\mathrm{a}}$ ). In the current study the interaction between HEIGHT and ASYM was explored. The combination of the two independent variables created six different load handling positions (Figure 7). To control the effect of the distance of the load on spinal loading, the straight line distance between the projected location of the load centre of mass and the centre of the subjects' ankles were kept constant at $45 \mathrm{~cm}$ across all conditions. Each subject performed a total of 24 trials (four repetitions for each of the 6 conditions) with the sequence completely randomized.

Three dependent variables were considered in the current study: 1. Increase in trunk flexion angle: the magnitude of increase in trunk flexion from initial trunk posture to the peak trunk flexion during the sudden impact; 2. Increase in L5/S1 joint moment: the difference between the initial joint moment and the maximum joint moment during the sudden impact; 3 . 
Peak L5/S1 joint compression force: the maximum spinal compressive force during the sudden loading event.

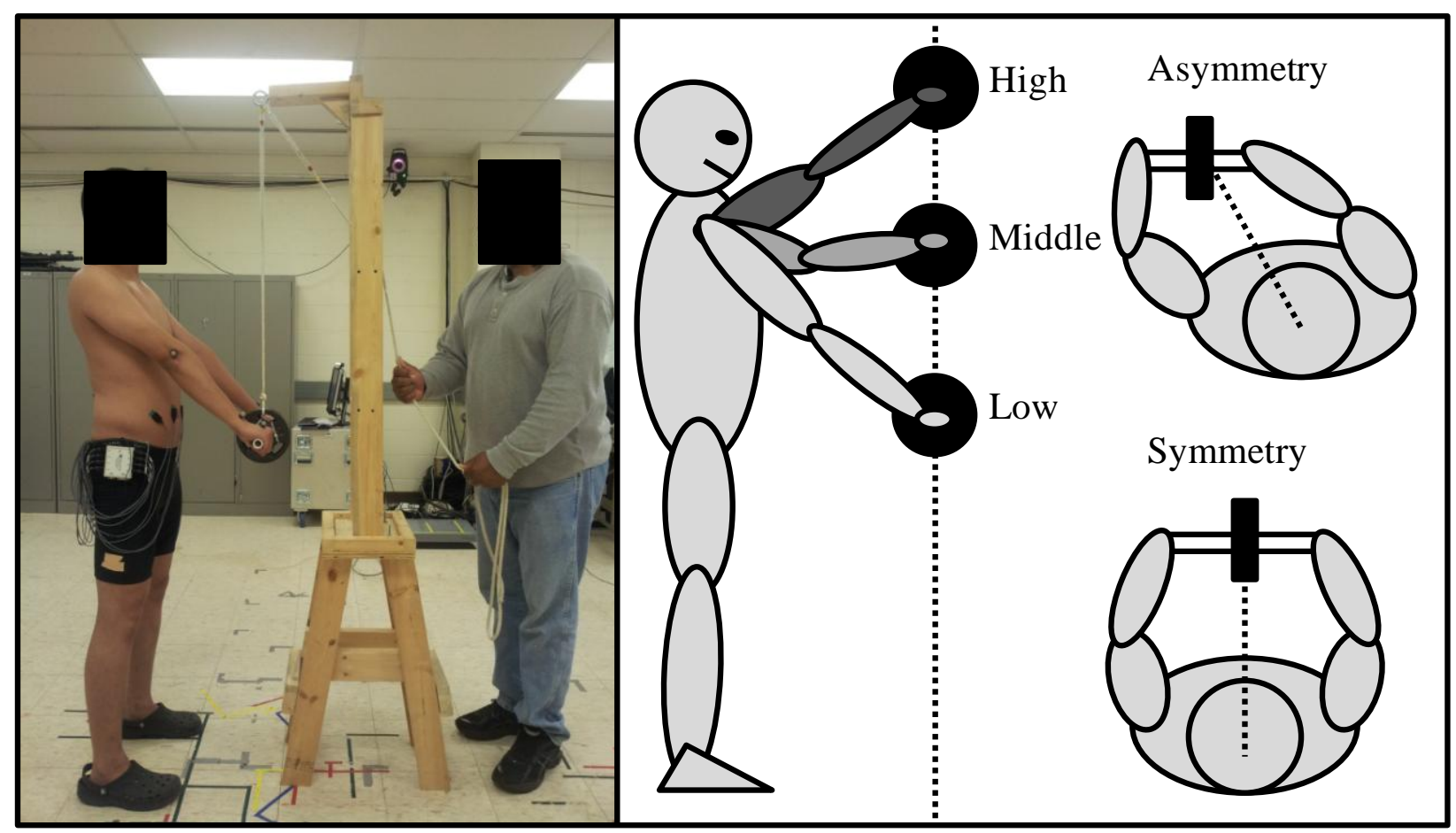

Figure 7. A side view of the experiment setup (left panel) and a demonstration of different load handling positions (right panel).

\subsubsection{Apparatus and Equipment}

A wood structure was built to hang the load at different height levels. The load was made from standard disc weights and secured through their centre holes using a polyvinyl chloride (PVC) pipe which also served as a handle for subjects to hold (Zhou et al. 2013 $3^{\mathrm{a}}$ ). The total weight of the load was $6.8 \mathrm{~kg}(15 \mathrm{lbs})$.

A surface electromyography (EMG) system (Model: Bagnoli, Delsys Inc, Boston, MA, USA) was used to record EMG signals at $1000 \mathrm{~Hz}$ from eight trunk muscles: left and right rectus abdominis (electrodes placed $2 \mathrm{~cm}$ above the umbilicus and $3 \mathrm{~cm}$ from the midline of the 
abdomen); left and right external obliques $(10 \mathrm{~cm}$ from the midline of the abdomen and $4 \mathrm{~cm}$ above the ilium with an angle of $45^{\circ}$ to the midline of the abdomen); left and right erector spinae ( $4 \mathrm{~cm}$ from the midline of $\mathrm{L} 3$ vertebra); left and right multifidus ( $2 \mathrm{~cm}$ from the midline of L4 vertebra). An eight camera (MX-13 series) 3D optical motion tracking system (Vicon, Nexus, Oxford, UK) was used to capture trunk and upper extremity kinematics at a sampling frequency of $100 \mathrm{~Hz}$. Eleven reflective markers were placed over the following positions: C7, T12, and L5 vertebrae; the most dorsal point of the acromioclavicular joint of the left and right shoulders; the most caudal point of the lateral epicondyle of the left and right elbows; the ulnar sides of the left and right wrists; the middle of the third metacarpal bone on both hands. Nexus 10.7 software (Vicon, Nexus, Oxford, UK) was used to record and synchronize both EMG and kinematics data. During the trunk muscle maximum voluntary contraction (MVC) trials, a lumbar dynamometer (Humac Norm, CSMi, MA, USA) and the attached back flexion-extension module were used to restrict subjects' pelvis and lower extremity and provide static resistance against trunk muscle maximum exertions.

\subsubsection{Procedure}

The experimental procedure was first explained to the subjects and the signed informed consent was obtained. A brief warm-up session was provided after the measurement of subjects' basic anthropometric data (body stature, mass, trunk length (from L5/S1 joint to the top of head), width (at iliac and xiphiod process levels) and depth (at iliac and xiphiod process levels)) to warm up muscles and have subjects practice the sudden loading tasks and familiarize with the experiment protocol. Then eight bi-polar EMG surface electrodes were attached over the skin of muscles of interest. When data collection started subjects first performed three repetitions of 
isometric maximum trunk extension/flexion exertions at 20 degree trunk flexion posture against a static resistance provided by the dynamometer while maintaining. Each MVC exertion lasted for 5 seconds and at least 2 minute of rest was provided to prevent muscle fatigue. EMG signals recorded during the MVC trials were later used to normalize the experimental EMG data.

Following the MVC trials, eleven reflective markers were fitted to the designated areas described above and subjects then performed all 24 experimental trials in a completely randomized order. During data collection subjects were instructed to stand straight and hold the load such that the distance between the projected location of the center of the load and the midpoint of the subjects' ankles remained constant $(45 \mathrm{~cm})$ among all conditions and trials (controlled by markers on the ground and assistance from experimenters). In the current study, elbow joint angles were not controlled in order to achieve designed postures. In the asymmetrical load position conditions, subjects rotated their arms leftward, kept their torso forward and maintained the same horizontal load distance as in the symmetrical conditions. In each trial, subjects stood with eyes closed, feet shoulder width apart. They were required to hold the load firmly, but without supporting the weight of the load; the load was then suddenly released by an experimenter without notice (Figure 7). Subjects were instructed to immediately arrest the falling load and carry it back to approximately its original position and hold it stably at this position for three seconds. At least 2 minutes of rest was provided between trials to prevent muscle fatigue.

\subsubsection{Data processing and analysis}

EMG signals were first filtered (band-pass filtered between 10 and $500 \mathrm{~Hz}$ and notch filtered at $60 \mathrm{~Hz}$ and its aliases) and rectified, then smoothed with a 200 data point ( 0.2 second) sliding window. EMG signals from all experimental trials were then normalized with respect to 
the maximum EMG activities collected during MVC trials for each muscle. The exported three dimensional coordinate data of the reflective markers were used to calculate trunk and upper extremity kinematics. The trunk flexion angle was defined as the angle formed by the line connecting the C7 and L5 reflective markers and the transverse plane (Ning et al. 2011).

\subsubsection{Biomechanical model}

In the present study, the external moment at the L5/S1 joint was estimated using a multisegment dynamic model in which seven body segments (trunk, left and right upper arm, forearm, and hand) and the external load were included. The mass and center of mass of all body segments were estimated according to previous work (Pheasant, 1986). A previously established and validated EMG-assisted biomechanical model (Marras and Granata, 1997) was used to calculate the muscle forces and the corresponding internal moment and spinal compression force at the L5/S1 joint. In this model, L5/S1 joint internal moment was estimated using equation (4), in which "Gaini", "NEMG ${ }_{i}$ " and " $A_{i}$ " represent muscle gain value, the normalized EMG and muscle cross-sectional area of trunk muscle $i$ respectively; " $f\left(l_{i}\right)$ " and " $f\left(v_{i}\right)$ " are the muscle forcelength and force-velocity modulation factors of muscle $i$ respectively (Davis et al. 1998; Marras and Granata, 1997), these five factors can be used to estimate force generated by muscle $i$. The additional factor " $r$ " is the moment arm vector of the trunk muscle $i$. The L5/S1 joint compression forces were estimated according to trunk muscle forces, trunk kinematics and geometry (e.g. line of action). The subject-specified moment arms $(r)$ and cross sectional areas (A) of the trunk muscles considered in the model were estimated using the predictive equations from earlier studies (Jorgensen et al. 2001; Marras et al. 2001). The maximum muscle stress 
(gain) value was then determined by matching the internal and external moments at the static load holding period (the last three seconds) of all trials.

$$
\vec{M}=\sum_{i}^{8} \vec{r}_{i} \times \operatorname{Gain}_{i} \times N E M G_{i} \times A_{i} \times f\left(l_{i}\right) \times f\left(v_{i}\right)
$$

\subsubsection{Statistical analysis}

A general linear model (as demonstrated below) was used to perform the statistical analysis.

$$
Y_{i j k}=\mu+\tau_{i}+\beta_{j}+\gamma_{k}+(\tau \beta)_{i j}+\varepsilon_{i j k l}
$$

Where $\mathrm{Y}_{\mathrm{ijk}}, \mu, \tau_{\mathrm{i}}, \beta_{\mathrm{j}}, \gamma_{\mathrm{k}}, \tau \beta_{\mathrm{ij}}$ and $\varepsilon_{\mathrm{ijkl}}$ represent biomechanical responses (dependent variables), overall mean, main effect of HEIGHT, main effect of ASYM, block effect, interaction effect between HEIGHT and ASYM, and random error. Total sum of squares was calculated as below:

$$
S S_{T}=\sum_{i=1}^{a} \sum_{j=1}^{b} \sum_{k=1}^{c} \sum_{l=1}^{n} Y_{i j k l}^{2}-\frac{y_{\ldots}^{2}}{a b c n}
$$

Sums of squares for the main effects and block effects were found as follows:

$$
S S A=\frac{1}{b c n} \sum_{i=1}^{a} y_{i \ldots}^{2}-\frac{y_{\ldots}^{2}}{a b c n}
$$




$$
\begin{aligned}
& S S B=\frac{1}{a c n} \sum_{j=1}^{b} y_{. j . .}^{2}-\frac{y_{\ldots .}^{2}}{a b c n} \\
& S S C=\frac{1}{a b n} \sum_{k=1}^{c} y_{. . . .}^{2}-\frac{y_{\ldots .}^{2}}{a b c n}
\end{aligned}
$$

Two factor interaction sum of squares was demonstrated as below:

$$
S S A B=\frac{1}{c n} \sum_{i=1}^{a} \sum_{j=1}^{b} y_{i j . .}^{2}-\frac{y_{\ldots \ldots}^{2}}{a b c n}-S S A-S S B
$$

Minitab statistical software (Minitab v.15. Inc., Pennsylvania, USA) was used for all statistical analyses. The assumptions of the ANOVA were first tested (Montgomery, 2005), and no violation of the assumptions was observed. Multivariate analyses of variance (MANOVAs) were performed to reveal the statistical significance of independent variables HEIGHT and ASYM as well as their interaction effect on all dependent variables. Variables with significant effects were then analyzed using repeated measures ANOVA with 'subject' considered as a blocking factor. Finally, Tukey-Kramer post-hoc tests were conducted on dependent variables that were significantly affected by HEIGHT to further investigate the differences between the two HEIGHT levels. The significant level of $\alpha$ was set as 0.05 for all statistical tests.

\subsection{Results}

Results of MANOVA revealed significant effects of HEIGHT, ASYM, and their interaction. Univariate ANOVA results demonstrated that both HEIGHT and ASYM 
significantly affected all three dependent variables. Only the increase of trunk flexion angle and L5/S1 joint moment were significantly influenced by the interactive effects of HEIGHT and ASYM (Table 3).

Table 3: The results of MANOVA and univariate ANOVA.

\begin{tabular}{lllll}
\hline & & \multicolumn{3}{c}{ ANOVA } \\
\cline { 3 - 5 } Independent Variables & MANOVA & $\begin{array}{l}\text { Trunk } \\
\text { angle }\end{array}$ & $\begin{array}{l}\text { L5/S1 } \\
\text { moment }\end{array}$ & $\begin{array}{l}\text { L5/S1 } \\
\text { compression }\end{array}$ \\
\hline HEIGHT & $\mathrm{P}<0.001$ & $\mathrm{P}<0.001$ & $\mathrm{P}<0.001$ & $\mathrm{P}<0.001$ \\
ASYM & $\mathrm{P}<0.001$ & $\mathrm{P}<0.001$ & $\mathrm{P}=0.004$ & $\mathrm{P}=0.009$ \\
HEIGHT*ASYM & $\mathrm{P}<0.001$ & $\mathrm{P}<0.001$ & $\mathrm{P}=0.002$ & $\mathrm{P}=0.25$ \\
\hline
\end{tabular}

The effects of HEIGHT on all dependent variables are demonstrated in Figure 8 to 10. A Significant increase of the peak L5/S1 joint compression force (on average from $2111 \mathrm{~N}$ to $2486 \mathrm{~N}$ ) was observed with an increase of load height (Figure 8, Different letters denote forces that are statistically different from one another. Bars indicate the corresponding $95 \%$ confidence interval). Reduced trunk flexion was also observed (on average from 6.5 degree to 3.8 degree) with an increase in load height (Figure 9, Different letters denote angles that are statistically different from one another. Bars indicate the corresponding 95\% confidence interval). Finally, significantly greater L5/S1 joint moment $(83.5 \mathrm{Nm})$ was observed at the 'Middle' height condition, while the difference between 'Low' and 'High' conditions was not statistically significant (Figure 10, Different letters denote moments that are statistically different from one another. Bars indicate the corresponding $95 \%$ confidence interval). 


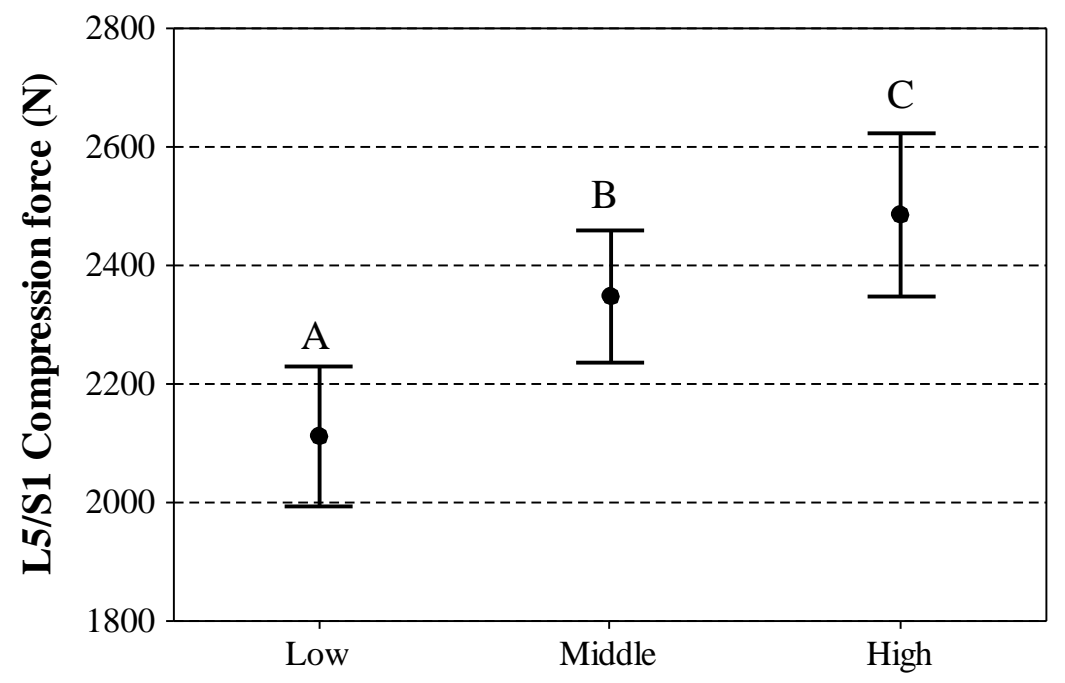

Figure 8. Peak L5/S1 compression force caused by sudden loading under the three different load height levels.

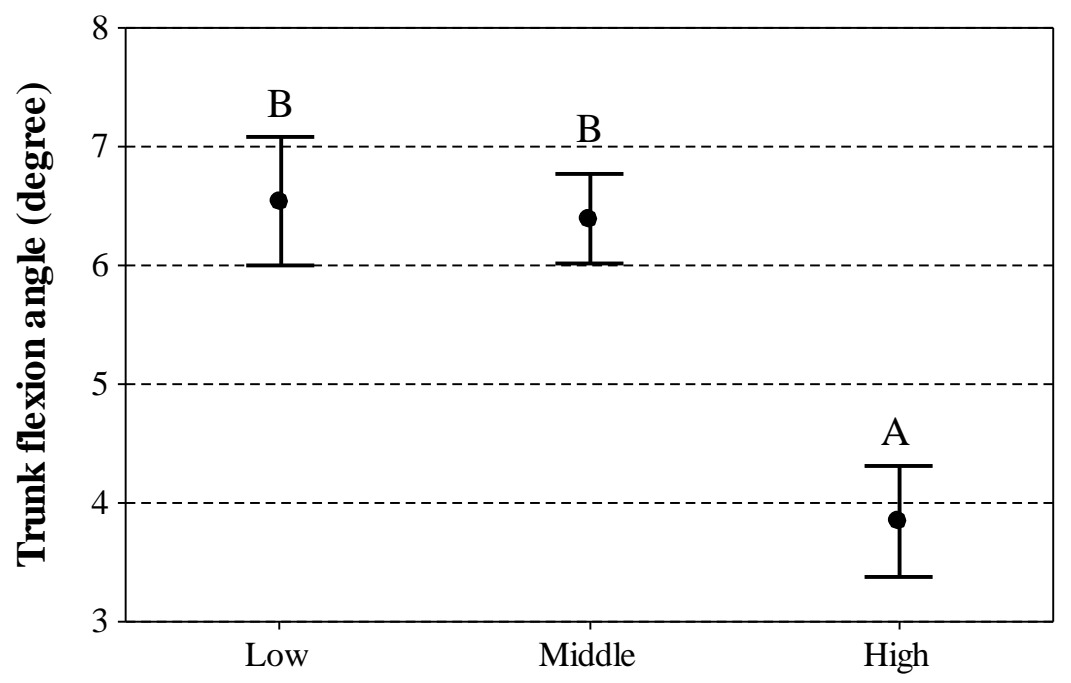

Figure 9. Increase of trunk flexion angle caused by sudden loading under the three different load height levels. 


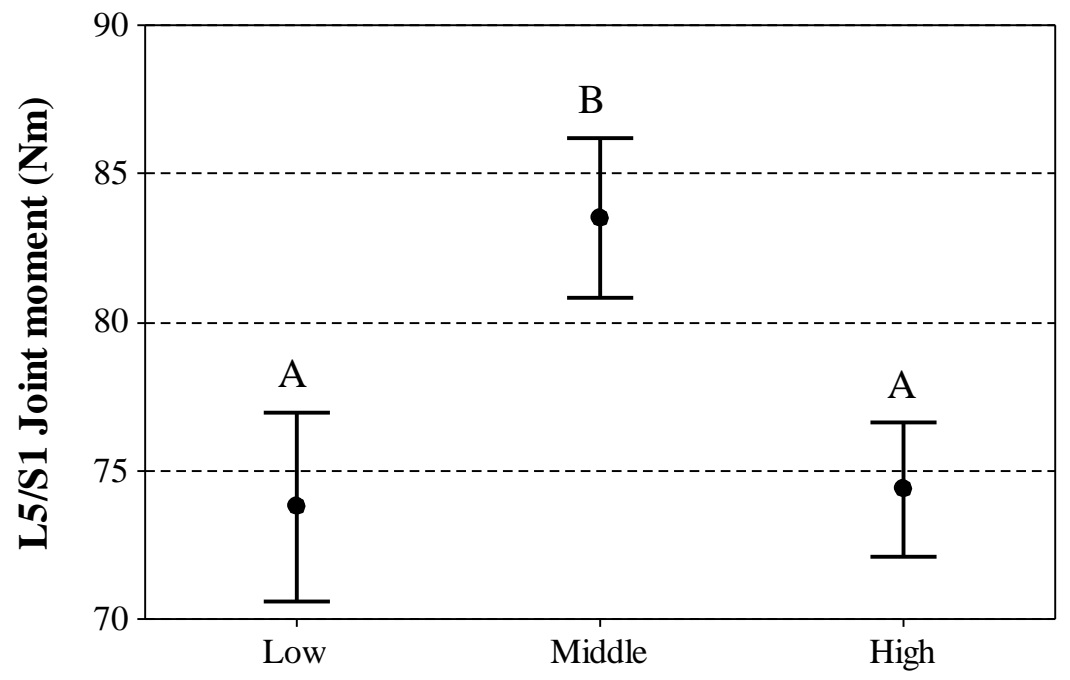

Figure 10. Increase of L5/S1 joint moment caused by sudden loading under the three different load height levels.

The effects of ASYM on all dependent variables are shown in Table 4. Significantly smaller increase of trunk flexion angle, L5/S1 moment, and peak L5/S1 joint compression force were found under the 'Asymmetry' condition. Furthermore, significant interaction effects between HEIGHT and ASYM for increase of trunk flexion angle and L5/S1 joint moment were observed (Figure 11 and Figure 12, Bars indicate the corresponding 95\% confidence interval). In the 'Symmetry' condition, the increase of load height resulted in smaller increase of trunk flexion. However, in the 'Asymmetry' condition the largest increase of trunk flexion was found in the 'Middle' condition. As showing in Figure 12, the largest L5/S1 joint moment was observed in the 'Middle' height condition in both ASYM conditions. However, the smallest L5/S1 joint moment were found in the 'High' and 'Low' levels in symmetric and asymmetric postures, respectively. These data also further indicated that the main effect of ASYM is primarily caused by the differences in the 'High' and 'Low' levels, but less by the variation in the 'Middle' level. 
Table 4. The mean (SD) values of dependent variables at different ASYM conditions, p-values are reported in Table 3.

\begin{tabular}{llll}
\hline ASYM & $\begin{array}{l}\text { Trunk angle } \\
\text { (degrees) }\end{array}$ & $\begin{array}{l}\text { L5/S1 moment } \\
(\mathrm{Nm})\end{array}$ & $\begin{array}{l}\text { L5/S1 } \\
\text { compression (N) }\end{array}$ \\
\hline Symmetry & $6.2(0.2)$ & $78.4(1.2)$ & $2360(56)$ \\
\hline Asymmetry & $5.0(0.2)$ & $76.0(1.2)$ & $2269(49)$ \\
\hline
\end{tabular}

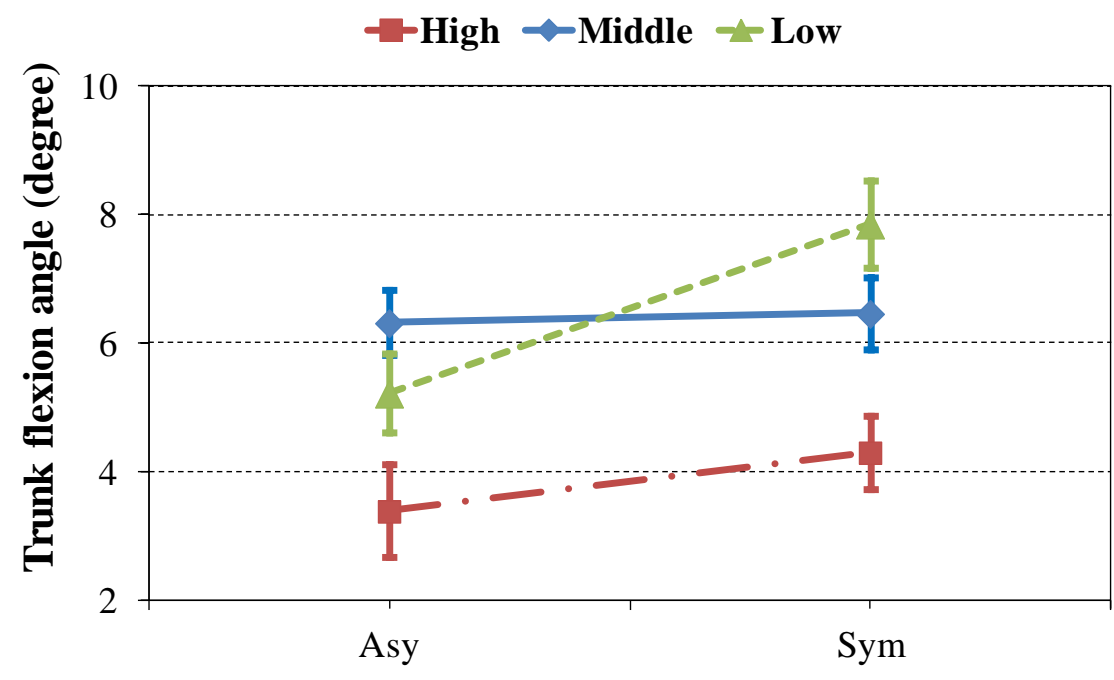

ASYM

Figure 11. Interaction between 'HEIGHT' and 'ASYM' for increase of trunk flexion angle. 


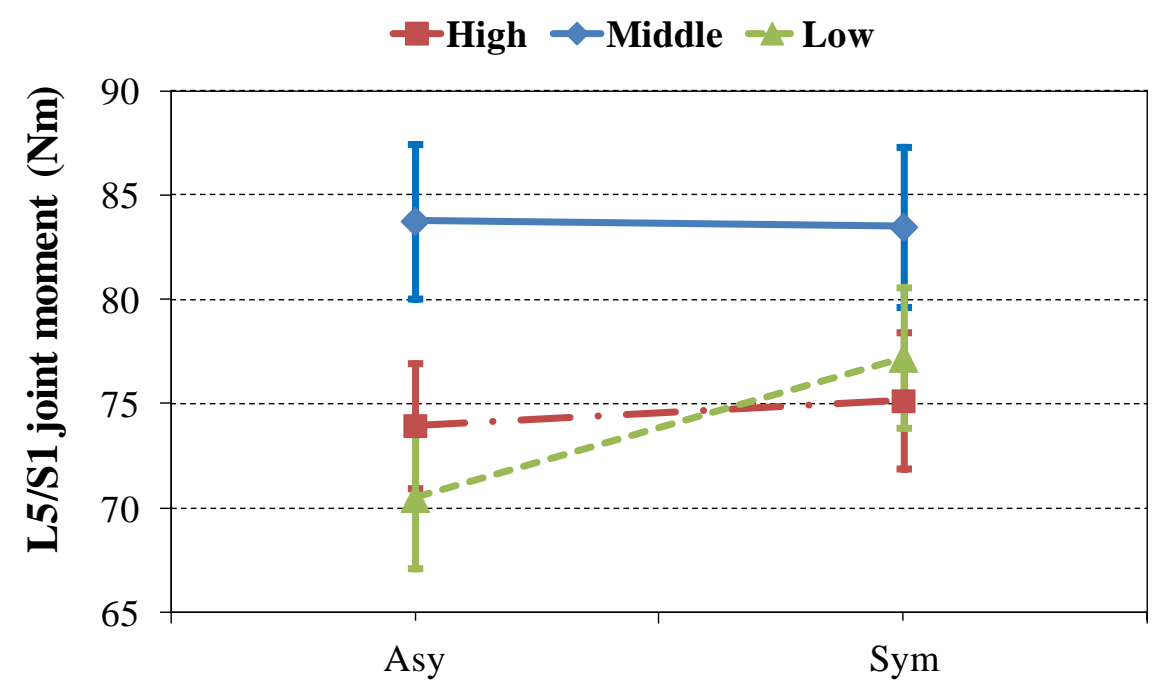

ASYM

Figure 12. Interaction between 'HEIGHT' and 'ASYM' for increase of L5/S1 joint moment.

In addition to the three dependent variables, several related kinematics variables were also analyzed. We found that Height significantly affected the magnitude of arm rotation (changes of sagittal angle from initial arm posture to the maximum downward arm rotation during sudden loading), initial trunk posture, initial load moment arm and the change of moment arm about L5/S1 joint (changes of loaf moment arm from initial posture to the point where peak moment was observed). As shown in Table 5 (different letters denote values that are significantly different from one another), significantly greater arm rotations were observed in the 'High' and 'Middle' height levels, compared with 'Low' level. Significantly smaller initial trunk flexion angle was observed in 'Low' than 'Middle' and 'High' height conditions. Initial load moment arm about L5/S1 joint was significantly smaller in 'High' level than the 'Middle' and 'Low' levels. During the sudden loading event, the peak L5/S1 joint moment occurred with reduced load moment arm (the load is closer to the body) in the "Low" level, but increased load moment arm (the load is further away from the body) in the 'Middle' and 'High' levels 
Table 5. The results of statistical analyses for related kinematics variables; mean (SD) values are shown in the table.

\begin{tabular}{ccccc}
\hline Height & $\begin{array}{c}\text { Arm rotation } \\
(\text { degrees })\end{array}$ & $\begin{array}{c}\text { Initial trunk } \\
\text { posture (degree) }\end{array}$ & $\begin{array}{c}\text { Initial moment arm } \\
(\mathrm{mm})\end{array}$ & $\begin{array}{c}\text { Change of moment } \\
\text { arm }(\mathrm{mm})\end{array}$ \\
\hline Low & $8.3(0.4)^{\mathrm{A}}$ & $91.7(0.4)^{\mathrm{A}}$ & $515.0(3.0)^{\mathrm{B}}$ & $-33.3(2.8)^{\mathrm{A}}$ \\
\hline Middle & $13.0(0.6)^{\mathrm{B}}$ & $95.0(0.3)^{\mathrm{B}}$ & $518.7(3.5)^{\mathrm{B}}$ & $20.4(1.9)^{\mathrm{B}}$ \\
\hline High & $12.4(0.8)^{\mathrm{B}}$ & $94.8(0.3)^{\mathrm{B}}$ & $487.5(2.9)^{\mathrm{A}}$ & $60.6(2.0)^{\mathrm{C}}$ \\
\hline$p$-value & $p<0.001$ & $p<0.001$ & $p<0.001$ & $p<0.001$ \\
\hline
\end{tabular}

To explain our results, muscle EMG activity data were also examined. Normalized EMG (NEMG with respect to MVC) data prior to the sudden loading event was first examined, and it was found that the average (SD) activities for erector spinae and multifidus are $4.8 \%(0.2 \%)$ and $4.1 \%(0.2 \%)$, respectively, which indicated that the back extensor muscles are relatively relaxed before the sudden loading event (Jin et al. 2012). In addition, the effect of HEIGHT on the trunk muscle peak MEMG during sudden loading was also investigated. Results demonstrated significantly elevated muscle activities among back extensor muscles and external oblique with the increase of HEIGHT (Figure 13, Different letters denote values that are statistically different from one another. Bars indicate the corresponding 95\% confidence interval, ES, MU, RA and EO refer to erector spinae, multifidus, rectus abdominis and external obliques, respectively). 


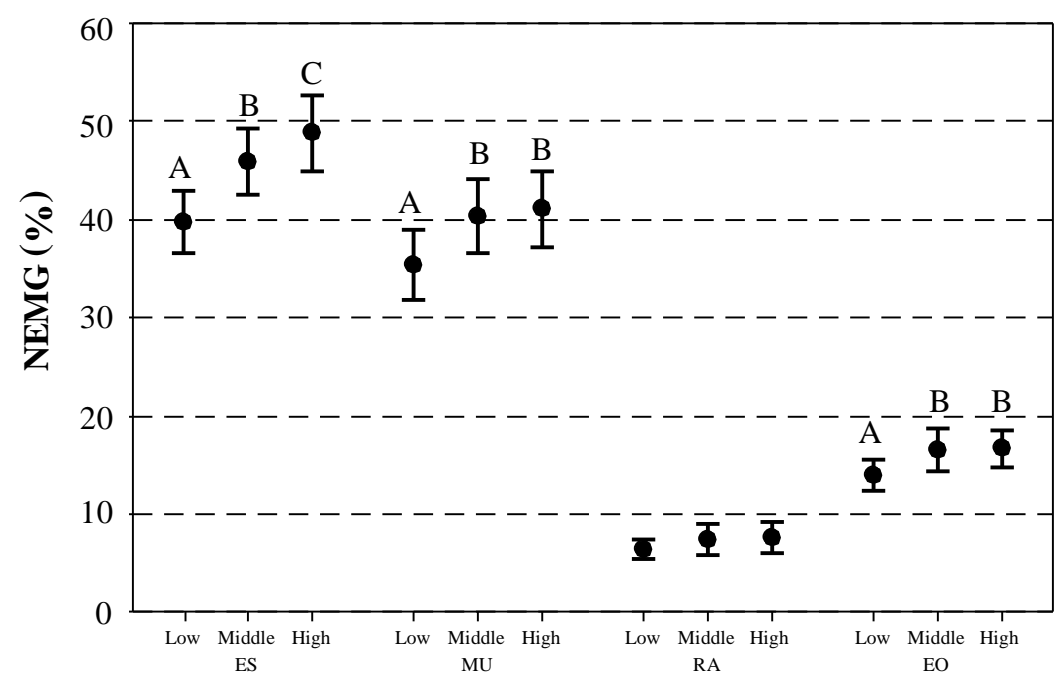

Figure 13. Peak normalized EMG value (average from both sides) of trunk muscles caused by sudden loading under the three different load height levels (two ASYM levels combined).

\subsection{Discussion}

In agreement with our initial hypotheses, results of the present study demonstrated increased spinal compression force and reduced trunk flexion at higher load handling position. These results can be explained by the stability and muscle co-contraction levels associated with different load handling height conditions. An earlier study demonstrated that the increase of load height elevated trunk muscle co-contraction during static weight holding due to reduced trunk stability (Granata and Orishimo, 2001); this increased trunk muscle activity would consequently increase spinal compression (Granata and Marras, 2000). Results of the current study supported the previous findings. Because of the strong positive association between spinal compression and the occurrence of low back injury (Kerr et al. 2001); our results indicated that handling load at lower height (i.e. the umbilicus level) helps reduce the risk of back injury when experiencing sudden loading during material handling. 
The increase of load handling height also resulted in smaller trunk flexion. As we have discussed above, the increase of load handling height may elevate the trunk muscle cocontraction (Granata and Orishimo, 2001), which results in higher global trunk stiffness, and smaller trunk bending under external impact (Granata and Marras, 2000). On the other hand, the magnitude of sagittal arm rotation may have also influenced trunk motion during sudden loading. As is shown in Table 5, significantly larger arm rotations were observed in the 'High' and 'Middle' height levels; such an increase in arm rotation may compensate for trunk motion and result in reduced trunk flexion.

In the current study the largest increase of L5/S1 joint moment was observed at the 'Middle' height level. According to the multi-segment model, the external moment on the L5/S1 joint is affected by the magnitude of external loads and their corresponding moment arms. Indepth analysis of kinematics data revealed that even though the horizontal distance between the load and the centre of ankles was controlled, subjects maintained slightly extended initial trunk postures when grasping load in the 'High' condition. This posture increased lumbar lordosis and decreased the initial moment arm between the L5/S1 joint and the load. Such reduction in moment arm could result in a smaller moment during sudden loading. In addition, although the initial moment arms of the external load were similar between 'Low' and 'Middle' levels, when releasing the load from the 'Low' position, the natural arm rotation brought the load closer to the human body which decreases the moment arm (an average decrease of $33.3 \mathrm{~mm}$ from the initial posture to the point that peak moment was observed) of the external load. In contrast, moment arms increased during sudden loading in both 'Middle' and 'High' levels (on average $60.6 \mathrm{~mm}$ and $20.4 \mathrm{~mm}$ increases from the initial posture to the point of peak moment respectively, as 
shown in Table 5). Consequently, the changes of moment arms in different conditions largely determined the results of the L5/S1 joint moment.

Results of this study demonstrated a significantly smaller increase of trunk flexion angle, L5/S1 joint moment, and peak L5/S1 joint compression force in the 'Asymmetry' load releasing position than the 'Symmetry' position (Table 4). These results were in agreement with the findings of a previous study (Zhou et al. 2013 ${ }^{\mathrm{a}}$ ). It has been suggested that asymmetric trunk posture increases torsional and lateral moment during lifting, therefore elevate the risk of back injury (Hooper et al. 1998). However, the asymmetric load handling posture tested in the current study did not involve trunk twisting motion (subjects were instructed to always face forward). Therefore, minor changes in spinal shear loading (both anterior-posterior and lateral shear forces) were generated due to the relatively small magnitude of posture perturbations (e.g. on average 5.0 degrees of trunk flexion). It should be noted that the current results only stand when handling relatively light load (e.g. $6.8 \mathrm{~kg}$ ) with the forward facing trunk postures. It is very likely that when handling a heavier load in flexed and/or rotated trunk postures, the sudden impact from an asymmetric direction could generate much higher torsional and shear spinal loading thereby elevates the risk of back injury.

Interestingly, a significant interaction effect between HEIGHT and ASYM was observed. In the 'Symmetry' condition, the increase of load height consistently reduced the magnitude of trunk flexion. However in the 'Asymmetry' condition the largest trunk flexion was found in the 'Middle' condition instead of in 'Low' condition. In addition, the largest L5/S1 joint moment was consistently found in the 'Middle' height condition in both ASYM conditions. However, the smallest L5/S1 joint moments were found in the 'Low' and 'High' conditions when holding the load in asymmetric and symmetric postures, respectively. In asymmetric load positions, the 
direction of external load and the sagittal plane formed a $45^{\circ}$ angle, which resulted in shorter moment arms to the rotation axis of sagittal trunk flexion than in symmetric conditions; therefore sudden loading in asymmetric conditions could generate less trunk flexion which was supported by the previous study (Zhou et al. $2013^{\mathrm{a}}$ ). In the current study this effect was more pronounced when holding the load at the 'Low' condition with lower trunk flexion angle and the resultant L5/S1 moment observed.

There are several noteworthy limitations of the present study. First, due to the relatively small trunk flexion during sudden loading, only spinal compression force was evaluated. More comprehensive spinal loading (compression force, shear force and torsional force etc.) should be assessed in future studies especially when experiencing larger trunk posture perturbations. Second, in the current study, the horizontal distance between the external load and the mid-point of the ankles was controlled to help maintain constant initial moment arm from the centre of the load to the L5/S1 joint. However this purpose was not attained due to the slight trunk hyperextension postures subjects maintained in higher load handling positions. Although our results are very unlikely to be caused by these changes (despite having the greatest spinal compression, the load moment arm in 'High' condition $(487.5 \mathrm{~mm})$ was even smaller than the 'Middle' and 'Low' conditions (518.7 and $515.0 \mathrm{~mm}$ respectively), but we observed the greatest spinal compression in the 'High' condition (Table 5)). Third, this study was conducted in a controlled lab environment with all subjects recruited from the student population, and a comparatively light load $(6.8 \mathrm{~kg})$ was used. The response of experienced workers to a much heavier load in a real work environment warrants future investigation. Forth, in the current study the asymmetric load position was created by rotating arms toward the left side of the body, yet the impact of handedness was not considered. 


\section{CHAPTER 5. THE STUDY OF THE EFFECT OF UNEVEN GROUND CONDITION}

\subsection{Objective}

In many occupational settings, workers have to perform manual material handling activities while standing on uneven ground surfaces, such as construction workers installing shingles on the roof, fisherman handling cables and cages on a rocking boat and farmers harvesting fruit and vegetable in an agricultural field. The purpose of the current experiment was to investigate the effects of laterally slanted ground on trunk biomechanical responses when experiencing sudden external loading. It is hypothesized that the increase of slanted ground angle will increase trunk muscle activation level and thereby generate higher L5/S1 joint compression force when experience sudden drop of load. Results of this study will facilitate the future development of guidelines and protective strategies to reduce occupational back injuries especially among workers in out-door occupations.

\subsection{Pilot study}

In the pilot study, two male subjects with mean (SD) height, weight, and age of 175.5 (3.5) $\mathrm{cm}, 72.5(3.5) \mathrm{kg}$, and 27.0 (1.4) years volunteered to participate. Both of them were recruited from the student population of West Virginia University and were free from low back pain and upper/lower extremity injuries.

The design of experiment involved two independent variables: laterally slanted angle (ANGLE) and weight of load (WEIGHT). Three different laterally slanted angles were tested: 0 degree (flat ground), 15 degree and 30 degree (demonstrated in Figure 14). Two different weights $(6.8 \mathrm{~kg}$ and $3.4 \mathrm{~kg})$ were used to investigate the interaction effect between slanted angle and the magnitude of the load. The combination of the two independent variables created 6 
conditions, and each condition was tested for 4 times (a total of 24 trials) in a completely randomized sequence. In all 6 testing conditions, the midpoint between the medial sides of ankles was superimposed onto the same location; the distance between this point and the projected center of mass of the external load was kept consistent for all conditions (Waters et al. 1993).

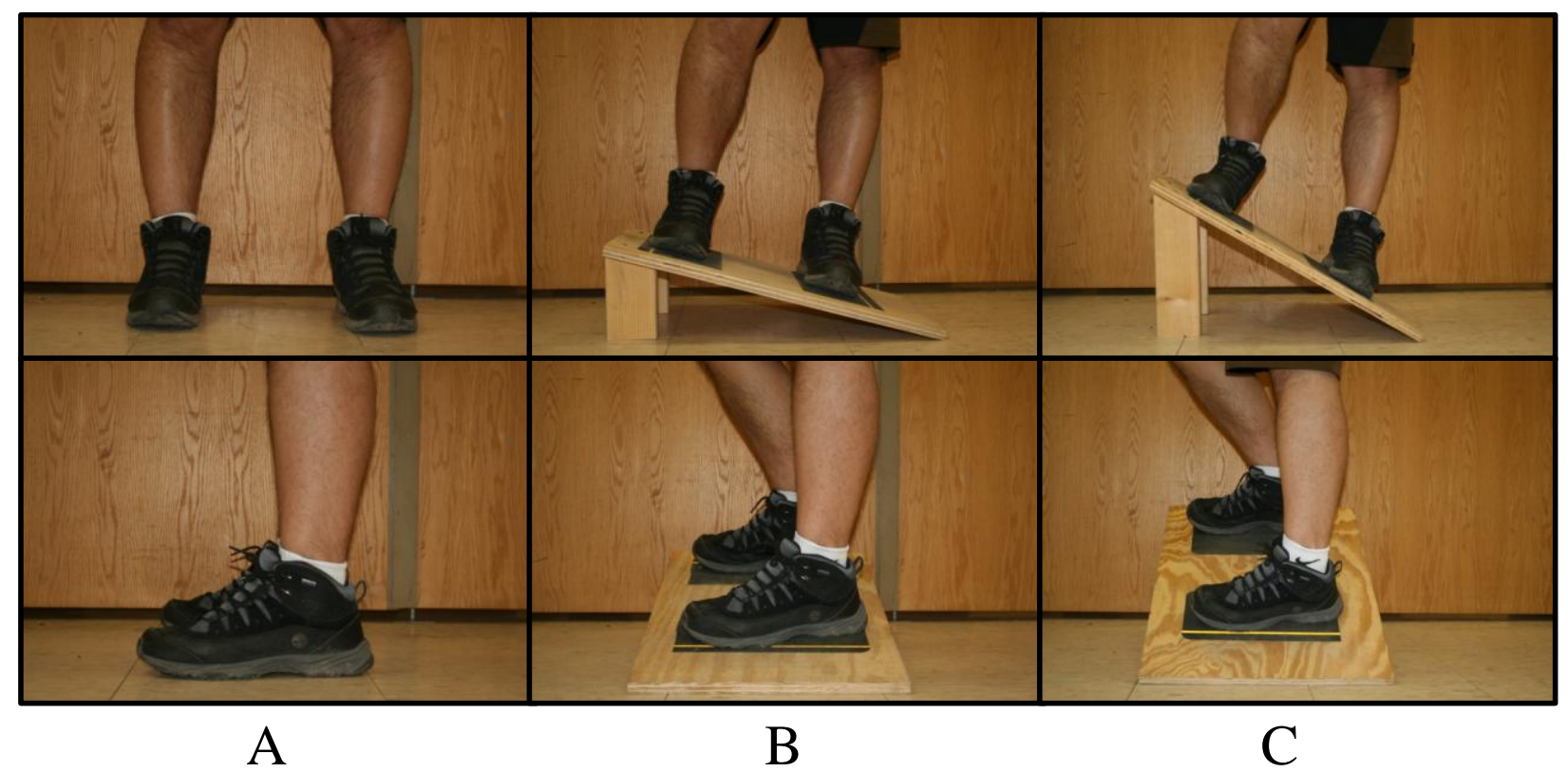

Figure 14. Three different ANGLE conditions (A: 0 degree; B: 15 degree; C: 30 degree).

In the pilot study, only one dependent variable was considered: Peak L5/S1 joint compression: the maximum spinal compressive force during the sudden impact.

This experiment used the same wood structure and load $(6.8 \mathrm{~kg})$ as the ones in previous experiments, and another load $(3.4 \mathrm{~kg})$ also made from standard disc weights and a polyvinyl chloride (PVC) pipe was included in the experiment to provide the other level of weight. Two customized wood slanted surfaces (15 degree and 30 degree) were built to provide the laterally 
slanted ground. Anti-skid strips were attached to the wood surfaces so as to have a higher coefficient of friction, and avoid the risk of falling.

The same electromyography (EMG) system, 3D optical motion tracking system (Vicon) and lumbar dynamometer were also used in this experiment to measure the activities of the major trunk muscles, capture trunk and upper extremity kinematics, and restrict subjects' pelvis and lower extremity and provide static resistance, respectively. The placements of EMG electrodes and Vicon reflective markers were the same as experiment 2.

Experiment procedure was first explained to the subjects and informed consents were obtained. Before the experiment, subjects' basic anthropometric data including body weight, height; trunk length, width and depth were measured, and a five minute warm-up routine was conducted to stretch and warm up trunk and upper extremity muscles. Subjects were then fitted with eight bi-polar EMG surface electrodes to the above mentioned sites and performed MVC trials in the back flexion-extension module. During the MVC, subjects were secured in a 20 degree trunk forward flexion posture and performed three repetitions of static trunk maximum extension/flexion exertions. Each MVC trial lasted five seconds and ample rest was given between trials in order to avoid muscle fatigue. The EMG activities of all sampled trunk muscles were recorded and used later as input to the biomechanical model.

Upon finishing the MVC trials, eleven reflective markers were secured to the designated locations and subjects performed all of the 24 experimental trials. In each trial, subjects first stood on the assigned slanted surface with feet shoulder width apart, and then held the load steadily (without supporting the load) with their eyes closed (Figure 15). The experimenter who controlled the load would suddenly release the load without notice. Subjects were trained to respond to the sudden loading by carrying the load back to approximately their shoulder level 
(the original load height) as quickly as possible, and holding the load stably for three seconds. Two minutes rest was given between trials to avoid the accumulation of muscle fatigue.

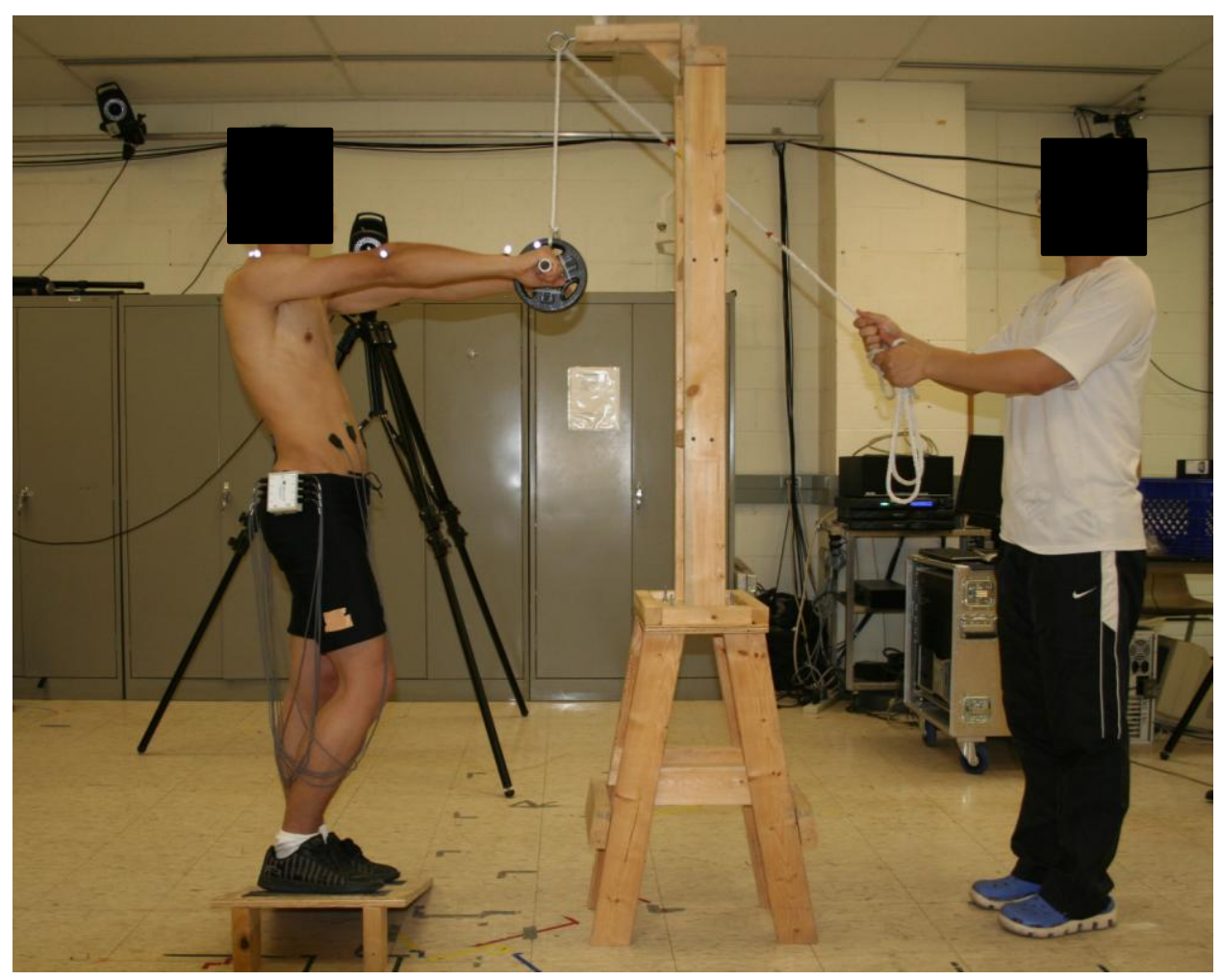

Figure 15. A side view of a sudden loading experimental trial: a subject is standing on slanted ground surface and holding the load before sudden loading event.

The EMG-assisted model, multi-segment dynamic model and their input parameters used in the previous experiments were also adopted in the current experiment to calculate the internal moment, spinal force and external moment at the L5/S1 joint.

Kinematics data captured by Vicon system and trunk muscle EMG activities recorded by EMG system were processed in the same manner as in the previous experiment. Trunk flexion angle, internal moment and spinal force at the L5/S1 joint were estimated in the same way as well. 
The results of the pilot study showed that peak L5/S1 joint compression force increased with a larger slanted angle (Figure 16), which is in line with our hypothesis. Also a heavier load resulted in larger peak L5/S1 compression force (Figure 17).

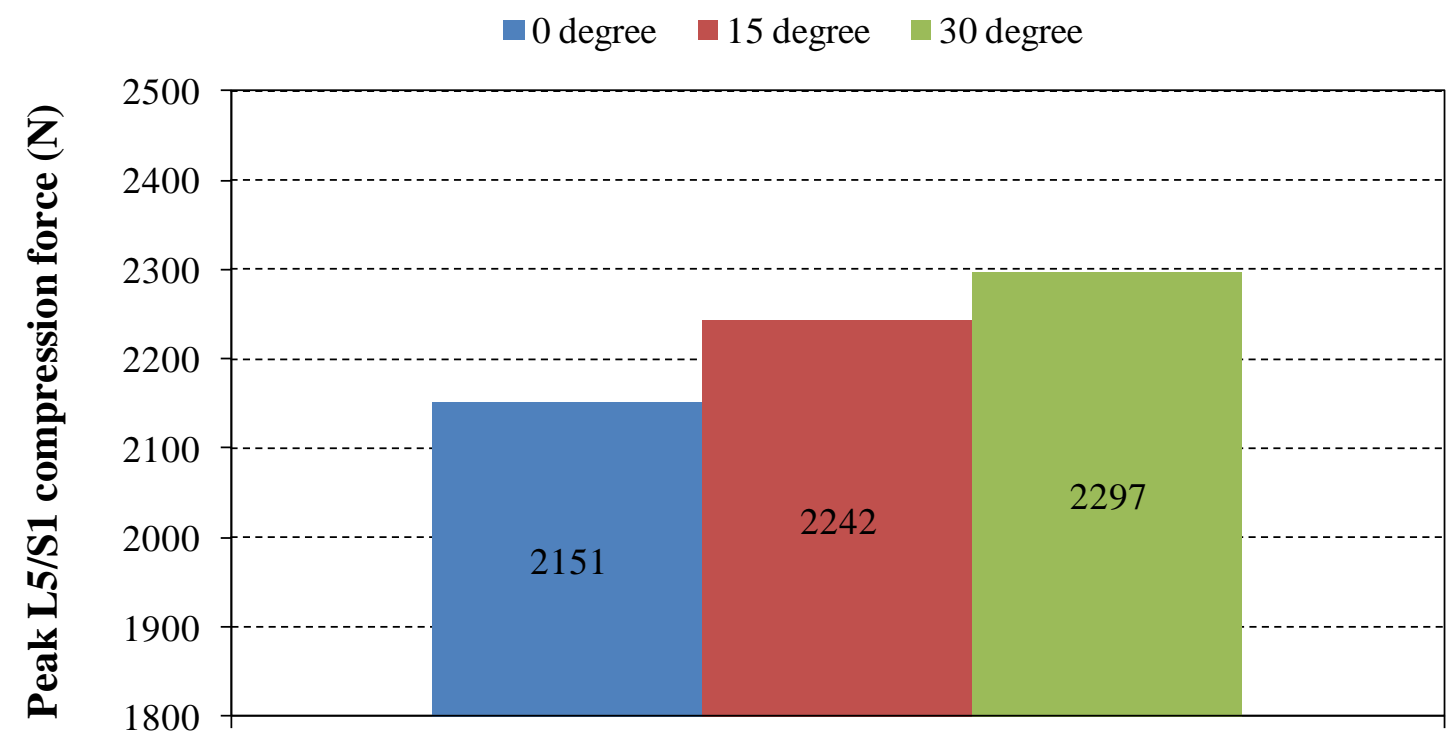

Figure 16. Peak L5/S1 compression force under three different slanted angle conditions.

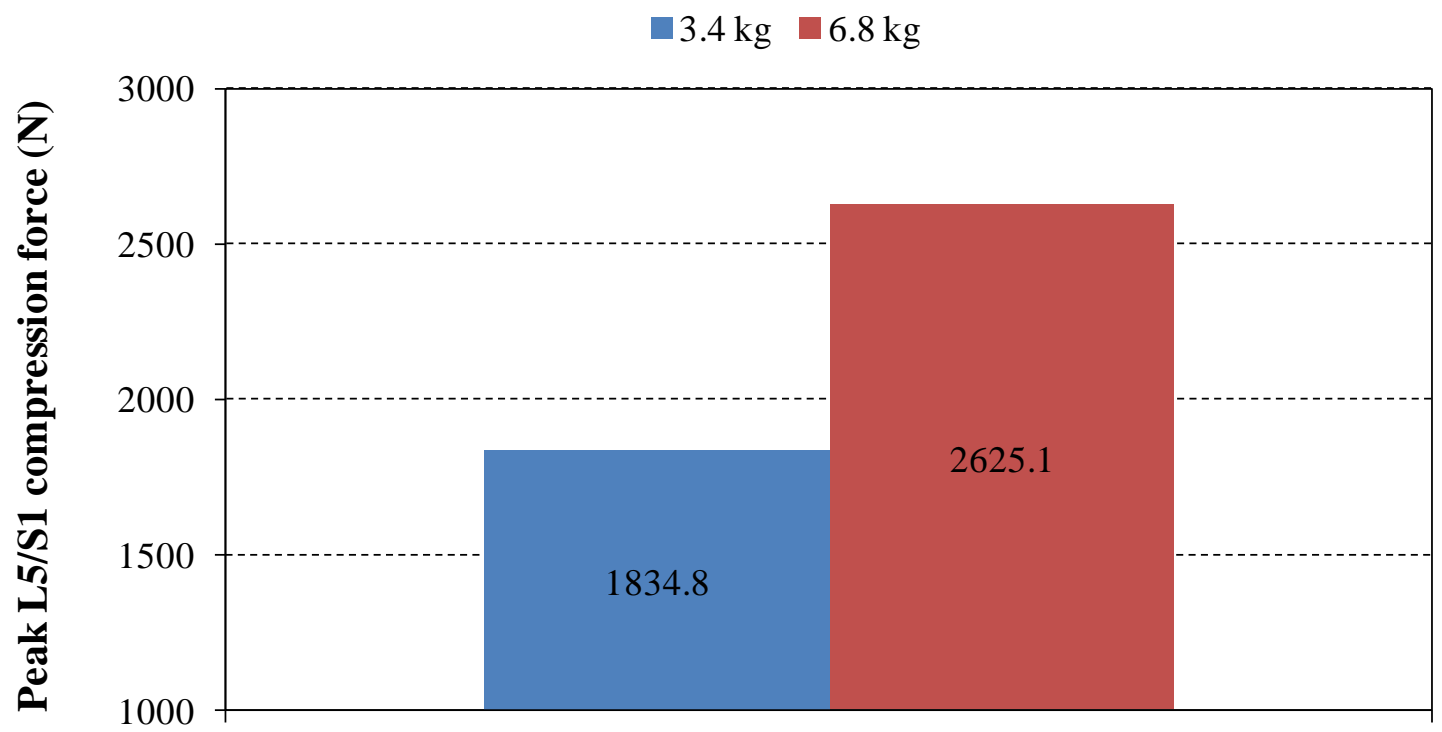

Figure 17. Peak L5/S1 compression force under three different weight conditions. 


\subsection{Method}

\subsubsection{Sample size}

The number of subjects was determined by the desired power of test and the results of pilot study, as demonstrated in the below equation (Dean and Voss. 1999):

$$
r=\frac{2 v \sigma^{2} \phi^{2}}{\Delta^{2}}
$$

Where $r, v, \sigma, \varphi$ and $\Delta$ is the desired number of observations, the number of levels of the treatment, the standard deviation of the response, the noncentrality parameter and the minimum difference in response beyond which the effects of the two treatments are considered significantly different from each other, respectively. And the procedure to find the required sample size is as follows (Dean and Voss):

1. Find the numerator degrees of freedom $v_{l}=v-1$, and specify $\alpha$ (usually $\alpha-0.01$ or 0.05 )

2. Specify the first iteration of the denominator degrees of freedom, and then calculate it using $v_{2}=v(r-1)$ in the following iterations

3. According to the desired power, use table (Power of F test table, see appendix 1) to determine $\varphi$ with $v_{l}$ and $v_{2}$ as inputs.

4. Calculate $r=2 v \sigma^{2} \phi^{2} / \Delta^{2}$, rounding to the nearest integer.

5. Repeat the above steps until $r$ is unchanged.

In the current study, $v$ is 3 ; the desired test power is 0.9 and $\alpha$ is 0.05 , respectively; and based on the result of the pilot study, $\sigma$ is 169.4 and 206.6 for $6.8 \mathrm{~kg}$ and $3.4 \mathrm{~kg}$ condition, respectively; $\Delta$ is $150 \mathrm{~N}$. According to Equation (5) and the aforementioned procedure, $r=27$ for 
$6.8 \mathrm{~kg}$ condition and $r=40$ for $3.4 \mathrm{~kg}$ condition, respectively. It was indicated that 10 subjects needed to be recruited, in order to detect a significant difference at the desired test power of 0.9. However, 13 subjects were finally recruited, such that a larger sample size could ensure an even higher test power and compensate for the possible overestimate of $\Delta$.

\subsubsection{Subjects}

In the current experiment, thirteen subjects from the student population of West Virginia University volunteered to participate in this study. All subjects were in good physical condition with average age, body height and body mass of 26.1 years (SD 2.3), $177 \mathrm{~cm}$ (SD 3.2) and 70.8 $\mathrm{kg}$ (SD 6.3), respectively, none had previous history of LBP or upper extremity injuries. The protocol of the experiment was approved by Research Integrity and Compliance Committee of West Virginia University.

\subsubsection{Experimental design}

The experimental design included two independent variables: laterally slanted angle (ANGLE) and weight of load (WEIGHT). ANGLE included three levels: 0 degree (flat ground), 15 degree and 30 degree (Figure 14). The effect of WEIGHT has already been investigated by a previous study (Zhou et al. 2013 ${ }^{\mathrm{b}}$ ), while in the current study, two different levels (6.8 $\mathrm{kg}$ and 3.4 $\mathrm{kg}$ ) of WEIGHT were also included to examine the interaction between ANGLE and WEIGHT. The two independent variables created 6 conditions $(3 \times 2)$, and each condition was repeated for 4 times, which formed a total of 24 trials. Across all experimental conditions, the distance between the midpoint of ankles and the projected center of mass of the external loads was kept at constant (subject specific arm length) (Waters et al. 1993). 
There were three dependent variables: 1 . Increase in trunk flexion angle: the difference between the peak trunk flexion angle and initial trunk angle; 2 . Increase in L5/S1 joint moment: the difference between the maximum L5/S1 joint moment during the sudden loading and initial L5/S1 joint moment; and 3. Peak L5/S1 joint compression force: the maximum L5/S1 joint compression forces during sudden loading.

\subsubsection{Apparatus and Equipment}

A wood structure was built to hang the loads at the shoulder level of subjects. The two loads were made from standard disc weights; a polyvinyl chloride (PVC) pipe was used to secure the weights together through the central holes, and to also serve as a handle for subjects to hold when performing the designated tasks. Two customized wood slanted surfaces (15 degree and 30 degree) were built to provide the laterally slanted ground. Anti-slip strips were attached to the wood surfaces so as to increase the coefficient of friction, and avoid the risk of falling.

A surface electromyography (EMG) system (Model: Bagnoli, Delsys Inc, Boston, MA, USA) was used to record EMG activities of four pairs of interested trunk muscles: left and right erector spinae (electrodes placed $4 \mathrm{~cm}$ to the midline at $\mathrm{L} 3$ vertebra); left and right multifidus (electrodes placed $2 \mathrm{~cm}$ to the midline at $\mathrm{L} 4$ vertebra); left and right rectus abdominis $(3 \mathrm{~cm}$ to the midline of the abdomen and $2 \mathrm{~cm}$ above the umbilicus); left and right external obliques (10 $\mathrm{cm}$ to the midline of the abdomen and $4 \mathrm{~cm}$ above the ilium with a $45^{\circ}$ angle to the midline of the abdomen).

An eight camera 3D optical motion tracking system (Model: MX-13 series, Vicon, Nexus, Oxford, UK) was used to capture the kinematics data of trunk and upper extremity. Reflective markers were attached on eleven landmarks of body segment: C7, T12, and L5 of 
vertebral column; left and right shoulders: the most dorsal point of the acromioclavicular; left and right elbows: the most caudal point of the lateral epicondyle; left and right wrists: the ulnar side of wrists; left and right hands: the middle of the third metacarpal bone on hands.

Besides, a lumbar dynamometer (Humac Norm, CSMi, MA, USA) and its attached back flexion-extension module were used in the maximum voluntary contraction (MVC) trials, so as to provide resistance to exert against and secure subjects' pelvis and lower limbs.

\subsubsection{Procedure}

At first, an experimenter explained the experimental procedure to the subjects, and then informed consents were signed. Subjects' basic anthropometric data (arm length, body mass, height, trunk length, width and depth) was measured prior to a five minute muscle warm-up session. Eight bi-polar EMG surface electrodes were placed over the skin of the above mentioned muscles and subjects then performed three repetitions of trunk MVC trials while maintain a 20 degree trunk forward flexion posture on the lumbar dynamometer (Ning et al. 2012). Each MVC exertion lasted for five seconds and two minutes rest was provided between trials to avoid muscle fatigue. The recorded EMG activities data during MVC was later used to normalize experimental EMG signals, and then served as input to the biomechanical model.

After completing the MVC trials, eleven reflective markers were fitted onto the above described locations of body segments, subjects then performed all 24 experimental trials. In each trial, subjects first stood on the assigned slanted surface with feet shoulder width apart, and then held the handle of the load steadily at their shoulder level without supporting the weight of the load. After subjects closed their eyes, an experimenter who controlled the load suddenly released the load without prior notice (Figure 15). Subjects were asked to hold the load and carry it back 
to approximately their shoulder level (the initial load height) as soon as possible, and hold it stably for three seconds. Two minutes of rest was given between sudden loading trials to prevent muscle fatigue.

\subsubsection{Biomechanical model}

The L5/S1 joint external moment was estimated by using a multi-segment dynamic motion model, in which the external loads and seven body segments (including trunk, upper arms, forearms and hands) were included. Trunk and upper extremities kinematics recorded by Vicon reflective markers as well as anthropometric data were used as inputs of this model, information about normalized mass (with respect to body mass) and the center of mass of the seven body segments was obtained from a previous study (Pheasant 1986). L5/S1 joint internal moment and spinal compression force were estimated using a previously established biomechanical model (Marras and Granata 1997). Input parameters (moment arms and the cross sectional areas of the trunk muscles) were estimated using regression equations established in previous studies (Jorgensen et al. 2001; Marras et al. 2001). The maximum muscle stress (gain) was determined by matching the external and internal L5/S1 joint moment during the static load holding phase (the last three seconds) of all experimental trials.

\subsubsection{Data processing and analysis}

Nexus 10.7 software was used to record and synchronize both EMG and kinematics data. Trunk flexion angle was defined as the angle between the transverse plane and the line connecting $\mathrm{C} 7$ and L5 reflective marker. EMG data were first filtered $(500 \mathrm{~Hz}$ low pass and $10 \mathrm{~Hz}$ high pass filter, notch filter of $60 \mathrm{~Hz}$ its aliases), rectified and smoothed (with a 200 data points 
sliding window). Then EMG signals of experimental trials were normalized (with respect to EMG data of MVC trials for each muscle) to provide NEMG data, which would be used later as inputs to the aforementioned biomechanical model to estimate L5/S1 joint internal moment and spinal compression force.

\subsubsection{Statistical analysis}

A general linear model was used to perform the statistical analysis (demonstrated below).

$$
Y_{i j k}=\mu+\tau_{i}+\beta_{j}+\gamma_{k}+(\tau \beta)_{i j}+\varepsilon_{i j k l}
$$

Where $\mathrm{Y}_{\mathrm{ijk}}, \mu, \tau_{\mathrm{i}}, \beta_{\mathrm{j}}, \gamma_{\mathrm{k}}, \tau \beta_{\mathrm{ij}}$ and $\varepsilon_{\mathrm{ijkl}}$ represent biomechanical responses (dependent variables), overall mean, main effect of ANGLE, main effect of WEIGHT, block effect, interaction effect between ANGLE and WEIGHT, and random error. Total sum of squares was calculated as below:

$$
S S_{T}=\sum_{i=1}^{a} \sum_{j=1}^{b} \sum_{k=1}^{c} \sum_{l=1}^{n} Y_{i j k l}^{2}-\frac{y_{\ldots .}^{2}}{a b c n}
$$

Sums of squares for the main effects and block effects were found as follows:

$$
S S A=\frac{1}{b c n} \sum_{i=1}^{a} y_{i \ldots}^{2}-\frac{y_{\ldots .}^{2}}{a b c n}
$$




$$
\begin{aligned}
& S S B=\frac{1}{a c n} \sum_{j=1}^{b} y_{. j .}^{2}-\frac{y_{\ldots .}^{2}}{a b c n} \\
& S S C=\frac{1}{a b n} \sum_{k=1}^{c} y_{. . k .}^{2}-\frac{y_{\ldots .}^{2}}{a b c n}
\end{aligned}
$$

Two factor interaction sum of squares was demonstrated as below:

$$
S S A B=\frac{1}{c n} \sum_{i=1}^{a} \sum_{j=1}^{b} y_{i j . .}^{2}-\frac{y_{\ldots \ldots}^{2}}{a b c n}-S S A-S S B
$$

The assumptions of the ANOVA (normality of residuals, non-correlation of residuals, and constant variance of residuals) were examined before analyses (Montgomery 2005), and no violation of the assumptions was observed. Multivariate analyses of variance (MANOVAs) were then performed to test the main and interaction effects. Variables that were found significant in the MANOVA were further analyzed using univariate ANOVA. Tukey-Kramer post-hoc tests were performed on the dependent variables that were significantly affected by ANGLE to further investigate the differences between levels. The $\alpha$-value of 0.05 was set in all statistical tests as the demand level of significance.

\subsection{Results}

Significant main effects of ANGLE and WEIGHT were found according to the results of MANOVA, while the interaction effect was not significant, and in thus was not further analysed. The follow up univariate ANOVA test revealed that ANGLE significantly affected the increase 
in L5/S1 joint moment and the peak L5/S1 joint compression force, and WEIGHT significantly affected all three dependent variables (Table 6).

Table 6. The results of MANOVA and univariate ANOVA.

\begin{tabular}{lllll}
\hline & & \multicolumn{3}{c}{ ANOVA } \\
\cline { 3 - 5 } Independent Variables & MANOVA & $\begin{array}{l}\text { Trunk } \\
\text { angle }\end{array}$ & $\begin{array}{l}\text { L5/S1 } \\
\text { moment }\end{array}$ & $\begin{array}{l}\text { L5/S1 } \\
\text { compression }\end{array}$ \\
\hline ANGLE & $\mathrm{P}<0.001$ & $\mathrm{P}=0.263$ & $\mathrm{P}<0.001$ & $\mathrm{P}<0.001$ \\
WEIGHT & $\mathrm{P}<0.001$ & $\mathrm{P}<0.001$ & $\mathrm{P}<0.001$ & $\mathrm{P}<0.001$ \\
ANGLE*WEIGHT & $\mathrm{P}=0.129$ & N/A & N/A & N/A \\
\hline
\end{tabular}

The effects of ANGLE on the three dependent variables are demonstrated in Figure 18 to 20. Greater increase of trunk flexion angle was caused by an increased slanted angle, while the effect was not significant (Figure 18).Significantly larger increase of L5/S1 joint moment and peak L5/S1 joint compression force (on average from $75.25 \mathrm{Nm}$ to $81.67 \mathrm{Nm}$ and from $2213 \mathrm{~N}$ to $2396 \mathrm{~N}$, respectively) were observed with the increase of slanted angle (Figure 19 and Figure 20, Different letters denote values that are statistically different from one another. Bars indicate the corresponding 95\% confidence interval.). 


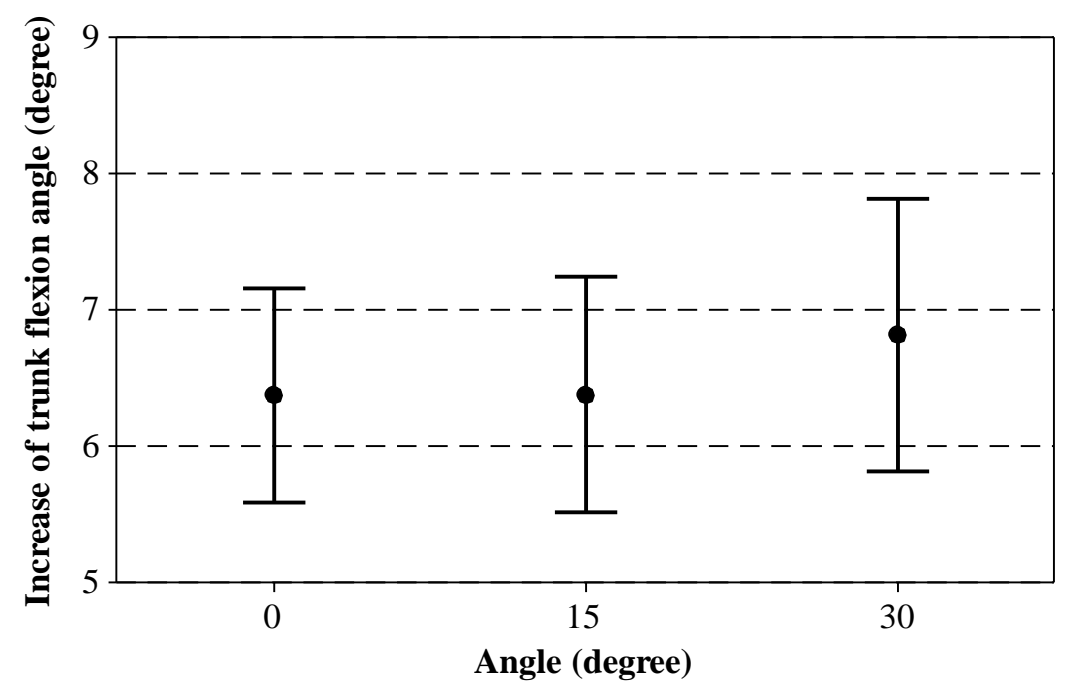

Figure 18. Increase of trunk flexion angle caused by sudden loading under the three different slanted angles.

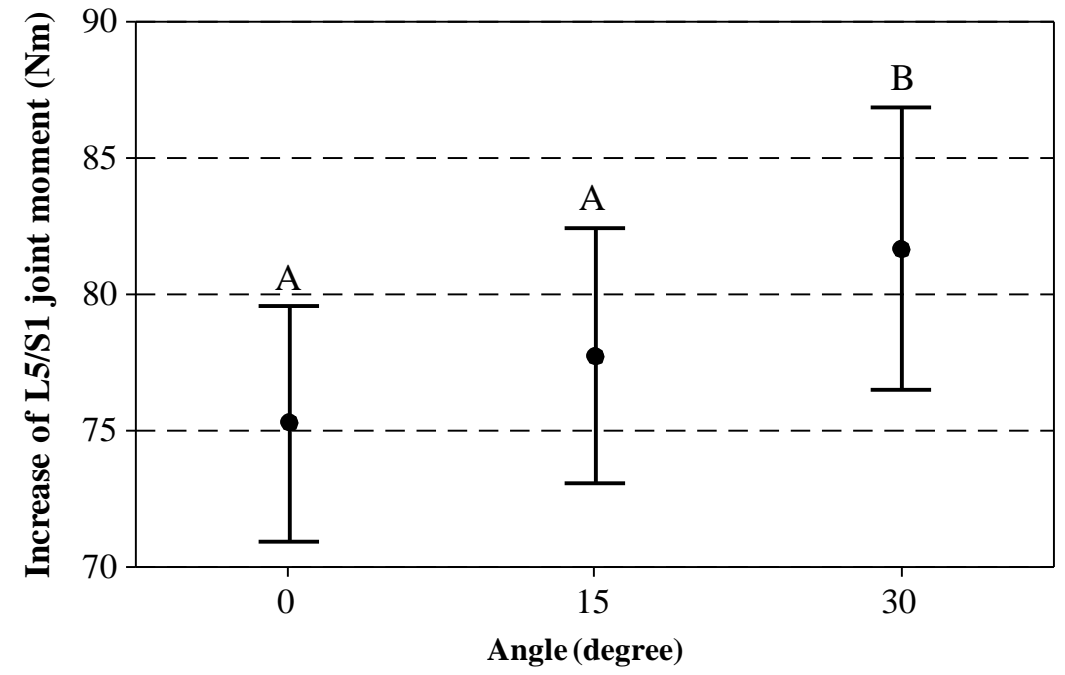

Figure 19. Increase of L5/S1 joint moment caused by sudden loading under the three different slanted angles. 


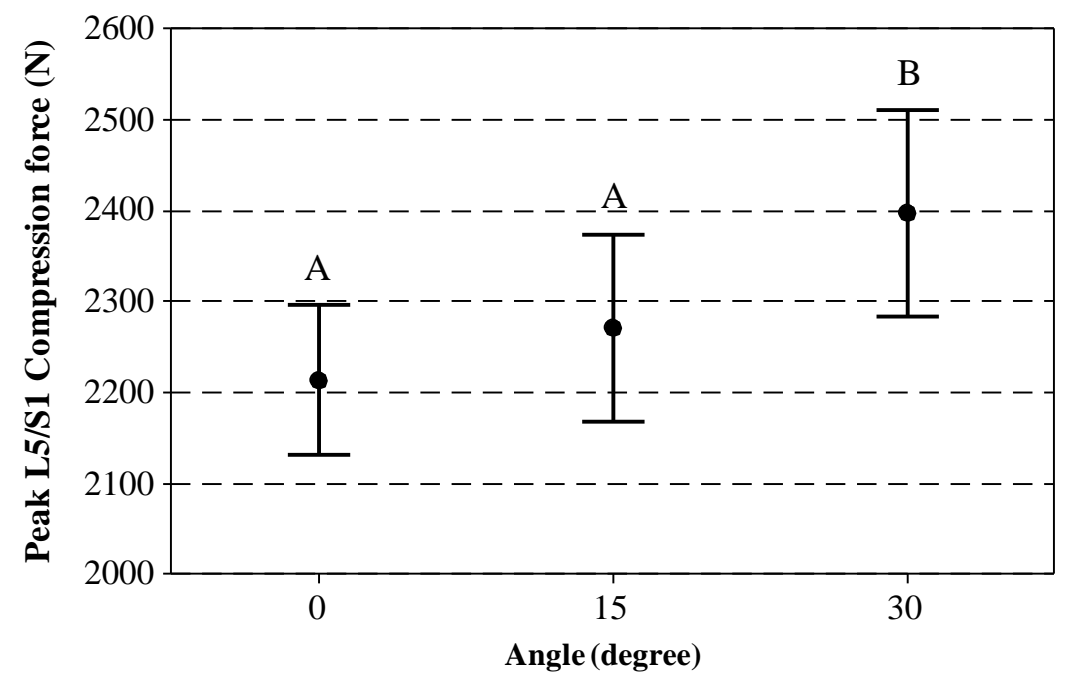

Figure 20. Peak L5/S1 compression force caused by sudden loading under the three different slanted angles.

The effects of WEIGHT on all three dependent variables are demonstrated in Table 7. Significantly smaller increase of trunk flexion angle, L5/S1 joint moment and peak L5/S1 joint compression force were found in the $3.4 \mathrm{~kg}$ weight condition.

Table 7. The mean (SD) values of dependent variables at different WEIGHT conditions, $p$-values are presented in Table 6.

\begin{tabular}{llll}
\hline WEIGHT & $\begin{array}{l}\text { Trunk angle } \\
\text { (degrees) }\end{array}$ & $\begin{array}{l}\text { L5/S1 moment } \\
(\mathrm{Nm})\end{array}$ & $\begin{array}{l}\text { L5/S1 } \\
\text { compression }(\mathrm{N})\end{array}$ \\
\hline $6.8 \mathrm{~kg}$ & $8.9(0.4)$ & $97.3(1.5)$ & $2675(32)$ \\
\hline $3.4 \mathrm{~kg}$ & $4.1(0.2)$ & $59.1(1.0)$ & $1911(24)$ \\
\hline
\end{tabular}

In addition, normalized EMG (NEMG) data (with respect to the MVC) of the eight trunk muscles were also examined. It was found that even though the differences of rectus abdominis muscles were not significant, with the increase of ANGLE, greater peak NEMG were generally observed from the antagonistic muscles (i.e. rectus abdominis and external obliques), which 
indicated higher co-contraction level of trunk muscles (Figure 21, RA and EO refer to rectus abdominis and external obliques, respectively. Different letters denote values that are statistically different from one another. Bars indicate the corresponding 95\% confidence interval). On the other hand, the NEMG of agonist muscles (i.e. erector spinae, multifidus) showed different pattern between left and right sides: the NEMG of the right (contralateral) side of both muscles increased with larger ANGLE; while for the left (ipsilateral) side, the smallest peak NEMG was constantly observed in 15 degree condition, while no significant difference was found between 0 and 30 degree conditions (Figure 22, ESl, ESr, MUl and MUr refer to left erector spinae, right erector spinae, left multifidus and right multifidus, respectively. Different letters denote values that are statistically different from one another. Bars indicate the corresponding $95 \%$ confidence interval).

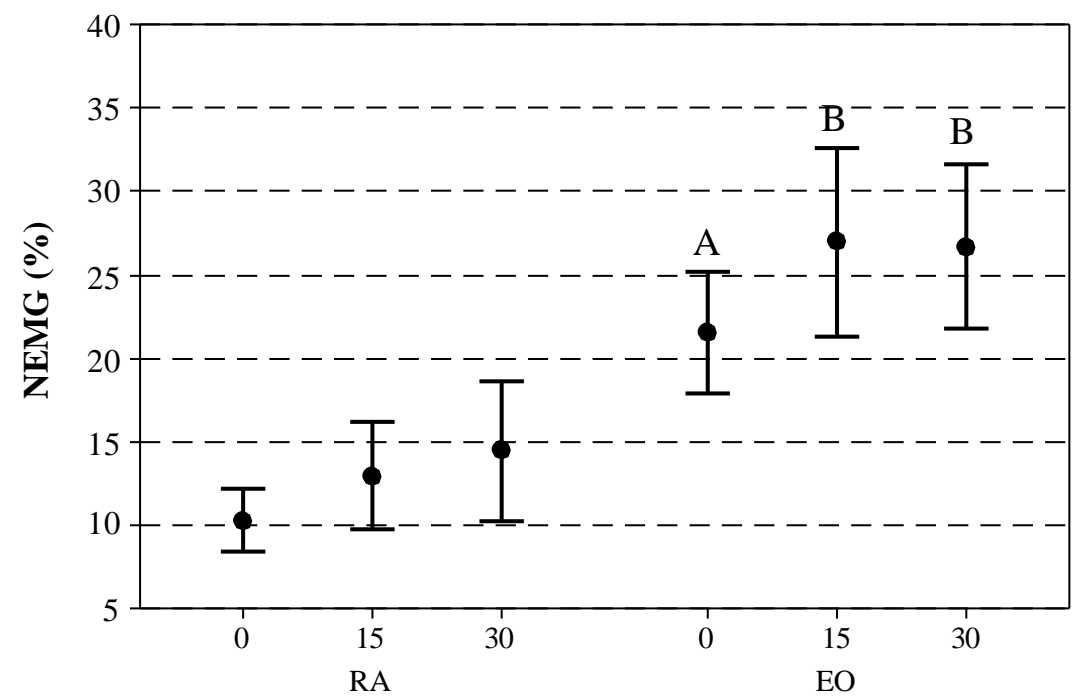

Figure 21. Averaged normalized EMG value (average of left and right sides with respect to MVC) of trunk antagonistic muscles. 


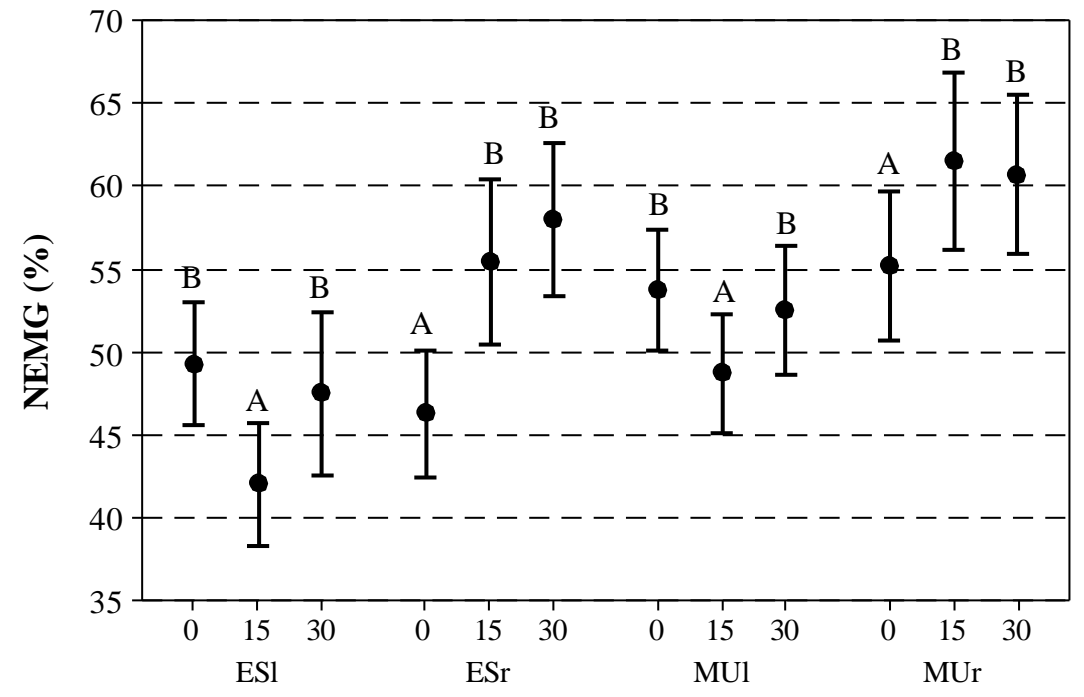

Figure 22. Normalized EMG pattern (with respect to MVC) of left and right sides of trunk agonist muscles.

\subsection{Discussion}

Results of the current study showed significant main effect of ANGLE on peak L5/S1 joint compression force, and post-hoc test revealed that an increase in ANGLE resulted in greater peak L5/S1 joint compression force, which confirmed our initial hypothesis. According to previous studies, an increase of slanted ground angle leads to reduced trunk stability, which in turn, elevates trunk muscles co-contraction (Granata and Orishimo, 2001) (supported by our NEMG data) and result in higher spinal compression force (Granata and Marras, 2000). Because spinal loading is directly associated with the risk of low back pain, to reduce such injury risk, slanted and uneven ground surfaces should be avoided in the work place especially when manual material handling tasks are performed on these surfaces. In the situation when uneven ground surface is inevitable, it is suggested that countermeasures that could compensate for the decreased postural stability should be taken. For example, roof workers should wear footwear that provides greater grip and friction. 
Attention should also be paid to the different NEMG patterns between the ipsilateral (left) and contralateral (right) sides of the agonist muscles. Due to the laterally unbalanced ground surface, the ipsilateral and contralateral sides of back muscles could generate substantially different biomechanical responses when performing different tasks. Such discrepancy has also been reported by previous studies. One study discovered different FRP onset patterns between left and right sides of trunk extensor muscles when performing trunk flexion/extension tasks while standing on laterally slanted ground ( $\mathrm{Hu}$ et al.2013). It was also found that when performing static load holding tasks on laterally slanted ground, the left and right sides of back muscles demonstrated considerably different EMG activities (Jiang et al. 2005). Consistent with these previous findings, in the current study different NEMG levels between left and right sides of trunk agonist muscles were observed during sudden loading trials when standing on laterally slanted ground surfaces. Because of the laterally slanted ground, subjects had their left leg straight while the knee of the right side flexed when performing sudden loading tasks. The flexion of knee could result in a forward pelvic rotation (Murray et al. 2002), which necessitate larger trunk muscle force to keep standing balance. Therefore, in the current study, with an increase in slanted ground angle, greater EMG activity was generally observed in contralateral side of trunk agonist muscles. On the other hand, the ipsilateral side did not show this pattern, with the increase of slanted ground angle (from 0 to 30 degree), EMG activity decreased first (from 0 to 15 degree), then significantly increased (from 15 to 30 degree). Similar result was reported in a previous study (Jiang et al. 2005), the activity of ipsilateral trunk extensor muscles reduced from 0 to 10 degree, while increased from 10 to 30 degree, the author presumed that this was due to a co-contraction strategy, which helped maintain torso stability. In 
the currently study, the reason why ipsilateral side of trunk extensor muscles showed this pattern is not clear, and warrants further analysis.

Even though the effect of ANGLE on the increase of trunk flexion angle was not significant, slightly larger increase of trunk flexion angle was observed in more slanted ground conditions. The increase of slanted angle reduced standing stability (Jiang et al. 2005), and therefore may result in greater trunk perturbation when subjects experience sudden loading.

The increase of slanged angle also significantly elevated the increase of L5/S1 joint moment during sudden loading. According to the multi-segments model, L5/S1 joint moment was highly influenced by trunk flexion angle as well as load moment arm. In the current study, as discussed above, even though the differences were not significant, greater increase of trunk flexion angle was still observed with the increase of ANGLE. This increase of trunk flexion angle partially contributed to the increase of L5/S1 joint moment. In addition, it was found in the present study that when standing on a more slanted ground surface, subjects tended to adopt a more flexed initial trunk posture. Such phenomenon supported the conclusions of a previous study that maintaining a more flexed trunk posture could compensate for the reduced stability (Granata and Wilson, 2001). While a more flexed trunk posture also resulted in larger moment arm between L5/S1 joint and the center of the load, which substantially increased the contribution of external load to the L5/S1 joint moment.

The results of the current study also showed a significant main effect of WEIGHT on all three dependent variables. Compared with $3.4 \mathrm{~kg}$ condition, greater trunk biomechanical responses were consistently generated in $6.8 \mathrm{~kg}$ condition, which was within our expectation and supported by a recent study (Zhou et al. 2013 ${ }^{\mathrm{b}}$ ). 
Several limitations of the current study need to be mentioned. First, participants of the current study were mostly college students with little experience in performing manual material handling tasks. More experienced workers may perform differently therefore warrant further investigation. Second, for safety reasons, only laterally slanted surfaces were tested and the largest slanted ground angle tested was 30 degrees, the biomechanical responses maybe different when standing on anterior posteriorly slanted surfaces and/or with larger slanted angles. 


\section{CHAPTER 6. CONCLUSION}

Findings of the current study provide important information regarding the influence of foot placement, load handling position and laterally slanted ground on trunk biomechanical responses during sudden external loading.

According to the results, it is suggested that adopting staggered stance could be a protective foot placement against low back injury for those who may experience sudden loading in their work environment.

In addition, handling load at a lower height (e.g. umbilicus) could help reduce the risk of low back injury as well; asymmetric load handling position generated by arm rotation resulted in smaller spinal compression force, while its impact on torsional and shear force need further investigation.

Finally, compared with flat ground, standing on laterally slanted ground surface will lead to increased spinal compression force, which indicates a higher risk of low back injury. Therefore, uneven ground condition, especially laterally slanted ground surface should be avoided in a workplace. Also, reducing the magnitude of impact load would reduce the risk of low back injury during sudden external loading.

As a primary contributing factor to low back injury, sudden loading warrants further investigations and more in depth understanding, such that more protective strategies can be developed. Future studies may investigate the effect of other work-related factors on people's biomechanical response during sudden loading, such as load coupling condition, flooring, upper extremities fatigue and restricted postures. Additionally, in the current study the sudden external loading was imposed on upper extremities, sudden loading of other types (applied on thoracic, pelvis and low extremities etc.) warrants future investigation. Last but not least, in the present 
study subjects experienced sudden loading while maintaining an upright standing posture, other postures (stoop, squat and kneeling, etc.) may generate a different response pattern, and should be studied in the future. 


\section{REFERENCES}

Andersson, G. B. (1981). Epidemiologic aspects on low-back pain in industry. Spine, 6(1), 5360.

Bakker, E. W., Verhagen, A. P., Lucas, C., Koning, H. J., de Haan, R. J., \& Koes, B. W. (2007). Daily spinal mechanical loading as a risk factor for acute non-specific low back pain: a case-control study using the 24-Hour Schedule. European Spine Journal, 16(1), 107-113.

BLS, 2012. Nonfatal Occupational Injuries and Illnesses Requiring Days Away from Work, 2011. Bureau of Labor Statistics.

Cholewicki, J., McGill, S. M., \& Norman, R. W. (1991). Lumbar spine loads during the lifting of extremely heavy weights. Medicine and science in sports and exercise, 23(10), 11791186.

Cholewicki, J., Panjabi, M. M., \& Khachatryan, A. (1997). Stabilizing Function of Trunk Flexor-Extensor Muscles Around a Neutral Spine Posture. Spine, 22(19), 2207-2212.

Chow, D. H., Cheng, A., Holmes, A. D., \& Evans, J. H. (2003). The effects of release height on center of pressure and trunk muscle response following sudden release of stoop lifting tasks. Applied ergonomics, 34(6), 611-619.

Davis, K. G., Marras, W. S., \& Waters, T. R. (1998). Evaluation of spinal loading during lowering and lifting. Clinical Biomechanics, 13(3), 141-152.

Dean, A. M., \& Voss, D. T. (1999). Design and analysis of experiments. Springer-Verlag, New York.

Drillis, R., Contini, R., \& Bluestein, M. (1964). Body segment parameters. Artificial limbs, 8(1), 44-66.

Druss, B. G., Marcus, S. C., Olfson, M., \& Pincus, H. A. (2002). The most expensive medical conditions in America. Health Affairs, 21(4), 105-111.

Frymoyer, J. W., \& Cats-Baril, W. L. (1991). An overview of the incidences and costs of low back pain. The Orthopedic Clinics of North America, 22(2), 263.

Granata, K. P., \& Marras, W. S. (2000). Cost-benefit of muscle cocontraction in protecting against spinal instability. Spine, 25(11), 1398-1404.

Granata, K. P., \& Orishimo, K. F. (2001). Response of trunk muscle coactivation to changes in spinal stability. Journal of Biomechanics, 34(9), 1117-1123. 
Granata, K. P., Slota, G. P., \& Wilson, S. E. (2004). Influence of fatigue in neuromuscular control of spinal stability. Human Factors: The Journal of the Human Factors and Ergonomics Society, 46(1), 81-91.

Granata, K. P., \& Wilson, S. E. (2001). Trunk posture and spinal stability. Clinical Biomechanics, 16(8), 650-659.

Greenwood, R., \& Hopkins, A. (1976). Muscle responses during sudden falls in man. The Journal of physiology, 254(2), 507-518.

Guo, H. R., Tanaka, S., Halperin, W. E., \& Cameron, L. L. (1999). Back pain prevalence in US industry and estimates of lost workdays. American Journal of Public Health, 89(7), 10291035.

Holbein-Jenny, M. A., McDermott, K., Shaw, C., \& Demchak, J. (2007). Validity of functional stability limits as a measure of balance in adults aged 23-73 years. Ergonomics, 50(5), 631-646.

Hooper, D. M., Goel, V. K., Aleksiev, A., Spratt, K., Bolte, K. M., \& Pope, M. (1998). Three dimensional moments in the lumbar spine during asymmetric lifting. Clinical Biomechanics, 13(6), 386-393.

Hoy, D., Bain, C., Williams, G., March, L., Brooks, P., Blyth, F., ... \& Buchbinder, R. (2012). A systematic review of the global prevalence of low back pain. Arthritis \& Rheumatism, 64(6), 2028-2037.

Jiang, Z., Shin, G., Freeman, J., Reid, S., \& Mirka, G. A. (2005). A study of lifting tasks performed on laterally slanted ground surfaces. Ergonomics, 48(7), 782-795.

Jin, S., Ning, X., \& Mirka, G. A. (2012). An algorithm for defining the onset and cessation of the flexion-relaxation phenomenon in the low back musculature. Journal of Electromyography and Kinesiology, 22(3), 376-382.

Jorgensen, M. J., Marras, W. S., Granata, K. P., \& Wiand, J. W. (2001). MRI-derived momentarms of the female and male spine loading muscles. Clinical Biomechanics, 16(3), 182193.

Kerr, M. S., Frank, J. W., Shannon, H. S., Norman, R. W., Wells, R. P., Neumann, W. P., ... \& Ontario Universities Back Pain Study Group. (2001). Biomechanical and psychosocial risk factors for low back pain at work. American journal of public health, 91(7), 1069.

Kirby, R. L., Price, N. A., \& MacLeod, D. A. (1987). The influence of foot position on standing balance. Journal of biomechanics, 20(4), 423-427. 
Lambeek, L. C., van Tulder, M. W., Swinkels, I. C., Koppes, L. L., Anema, J. R., \& van Mechelen, W. (2011). The trend in total cost of back pain in The Netherlands in the period 2002 to 2007. Spine, 36(13), 1050-1058.

Lavender, S. A., \& Marras, W. S. (1995). The effects of a temporal warning signal on the biomechanical preparations for sudden loading. Journal of Electromyography and Kinesiology, 5(1), 45-56.

Lavender, S. A., Marras, W. S., \& Miller, R. A. (1993). The development of response strategies in preparation for sudden loading to the torso. Spine, 18(14), 2097-2105.

Lavender, S. A., Mirka, G. A., Schoenmarklin, R. W., Sommerich, C. M., Sudhakar, L. R., \& Marras, W. S. (1989). The effects of preview and task symmetry on trunk muscle response to sudden loading. Human Factors: The Journal of the Human Factors and Ergonomics Society, 31(1), 101-115.

Lawrence, B. M., Mirka, G. A., \& Buckner, G. D. (2005). Adaptive system identification applied to the biomechanical response of the human trunk during sudden loading. Journal of biomechanics, 38(12), 2472-2479.

Maetzel, A., \& Li, L. (2002). The economic burden of low back pain: a review of studies published between 1996 and 2001. Best Practice \& Research Clinical Rheumatology, $16(1), 23-30$.

Manning, D. P., Mitchell, R. G., \& Blanchfield, L. P. (1984). Body movements and events contributing to accidental and nonaccidental back injuries. Spine, 9(7), 734-739.

Marras, W. S. (2000). Occupational low back disorder causation and control. Ergonomics, 43(7), 880-902.

Marras, W. S., \& Granata, K. P. (1997). The development of an EMG-assisted model to assess spine loading during whole-body free-dynamic lifting. Journal of Electromyography and Kinesiology, 7(4), 259-268.

Marras, W. S., Jorgensen, M. J., Granata, K. P., \& Wiand, B. (2001). Female and male trunk geometry: size and prediction of the spine loading trunk muscles derived from MRI. Clinical Biomechanics, 16(1), 38-46.

Marras, W. S., Rangarajulu, S. L., \& Lavender, S. A. (1987). Trunk loading and expectation. Ergonomics, 30(3), 551-562.

Mawston, G. A., McNair, P. J., \& Boocock, M. G. (2007). The effects of prior exposure, warning, and initial standing posture on muscular and kinematic responses to sudden loading of a hand-held box. Clinical Biomechanics, 22(3), 275-281. 
McCoy, C. E., Hadjipavlou, A. G., Overman, T., Necessary, J. T., \& Wolf, C. (1997). Workrelated low back injuries caused by unusual circumstances. Journal of Orthopaedic \& Sports Physical Therapy, 26(5), 260-265.

McGill, S. M. (1997). The biomechanics of low back injury: implications on current practice in industry and the clinic. Journal of biomechanics, 30(5), 465-475.

Montgomery, D. C., 2005. Design and analysis of experiments, Sixth ed. John Wiley \& Sons, New York, NY.

Murray, R., Bohannon, R., Tiberio, D., Dewberry, M., \& Zannotti, C. (2002). Pelvifemoral rhythm during unilateral hip flexion in standing. Clinical Biomechanics, 17(2), 147-151.

Ning, X., Haddad, O., Jin, S., \& Mirka, G. A. (2011). Influence of asymmetry on the flexion relaxation response of the low back musculature. Clinical Biomechanics, 26(1), 35-39.

Ning, X., Jin, S., \& Mirka, G. A. (2012). Describing the active region boundary of EMG-assisted biomechanical models of the low back. Clinical Biomechanics, 27(5), 422-427.

Ning, X., \& Mirka, G. A. (2010). The effect of sinusoidal rolling ground motion on lifting biomechanics. Applied ergonomics, 42(1), 131-137.

Ning, X., Zhou, J., Dai, B., \& Jaridi, M. (2014). The assessment of material handling strategies in dealing with sudden loading: The effects of load handling position on trunk biomechanics. Applied ergonomics.

Norman, R., Wells, R., Neumann, P., Frank, J., Shannon, H., \& Kerr, M. (1998). A comparison of peak vs cumulative physical work exposure risk factors for the reporting of low back pain in the automotive industry. Clinical Biomechanics, 13(8), 561-573.

Omino, K., \& Hayashi, Y. (1992). Preparation of dynamic posture and occurrence of low back pain. Ergonomics, 35(5-6), 693-707.

Pheasant, S., 1986. Bodyspace: Anthropometry, Ergonomics and Design, Taylor \& Francis, London, pp, 131-133.

Sorensen, C. J., Haddad, O., Campbell, S., \& Mirka, G. A. (2011). The effect of stance width on trunk kinematics and trunk kinetics during sagitally symmetric lifting. International Journal of Industrial Ergonomics, 41(2), 147-152.

Stewart, W. F., Ricci, J. A., Chee, E., Morganstein, D., \& Lipton, R. (2003). Lost productive time and cost due to common pain conditions in the US workforce. Jama, 290(18), 24432454. 
Stokes, I. A., Gardner-Morse, M., Henry, S. M., \& Badger, G. J. (2000). Decrease in trunk muscular response to perturbation with preactivation of lumbar spinal musculature. Spine, 25(15), 1957-1964.

Walker, B. F. (2000). The prevalence of low back pain: a systematic review of the literature from 1966 to 1998. Journal of Spinal Disorders \& Techniques, 13(3), 205-217.

Waters, T. R., Putz-Anderson, V., Garg, A., \& Fine, L. J. (1993). Revised NIOSH equation for the design and evaluation of manual lifting tasks. Ergonomics, 36(7), 749-776.

Webster, B. S., \& Snook, S. H. (1994). The cost of 1989 workers' compensation low back pain claims. Spine, 19(10), 1111-1115.

Yelin, E., \& Callahan, L. F. (1995). Special article the economic cost and social and psychological impact of musculoskeletal conditions. Arthritis \& Rheumatism, 38(10), 1351-1362.

Zedka, M., Prochazka, A., Knight, B., Gillard, D., \& Gauthier, M. (1999). Voluntary and reflex control of human back muscles during induced pain. The Journal of physiology, 520(2), 591-604.

Zhou, J., Dai, B., \& Ning, X. (2013). The assessment of material handling strategies in dealing with sudden loading: influences of foot placement on trunk biomechanics. Ergonomics, 56(10), 1569-1576.

Zhou, J., Ning, X., \& Dai, B. (2013, September). Trunk Kinematics under Sudden Loading Impact when Adopting Different Foot Postures. In Proceedings of the Human Factors and Ergonomics Society Annual Meeting (Vol. 57, No. 1, pp. 929-933). SAGE Publications. 


\section{Appendix A: CONSENT AND INFORMATION FORM}

\section{West VirginiaUniversity. \\ Office of Research Compliance \\ CONSENT AND INFORMATION FORM}

\section{OMR ICF}

Principal Investigator: Ning, Xiaopeng

Department: $\quad$ ENGINEERING - Ind./Mgt. Sys. Engineering

Tracking Number: $\quad \mathrm{H}-24416$

\section{Study Title:}

The effect of foot positioning on the trunk biomechanical responses of sudden external loading.

Co-Investigator(s):

,Zhou, Jie, Hu, Boyi,

\section{Sponsor}

\section{Contact Persons}

In the event you experience any side effects or injury related to this research, you should contact Dr. Xiaopeng Ning at 304/ 294-9474. (After hours contact Dr. Xiaopeng Ning at 515/520-1951.) If you have any questions, concerns, or complaints about this research, you can contact Dr. Xiaopeng Ning or Mr. Jie Zhou at 304/294-9474.

For information regarding your rights as a research subject, you may contact the Office of Research Compliance at 304/293-7073.

\section{Introduction}

In addition if you would like to discuss problems, concerns, have suggestions related to research, or would like to offer input about the research, contact the Office of Research Integrity and Compliance at 304293-7073.You, have been asked to participate in this research study, which has been explained to you by Mr.

\begin{tabular}{lll}
\hline Tracking \#: & $\mathrm{H}-24416$ & Page 1 of 5 \\
Approved On: & $10 / 23 / 2012$ & \\
Valid Through: & $10 / 22 / 2013$ & \\
Last Amended: & $\mathrm{N} / \mathrm{A}$ &
\end{tabular}

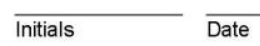


Tracking \#: H-24416

Jie Zhou. This study is conducted by Xiaopeng Ning (PhD), Jie Zhou (BS) and Boyi Hu (MS) in the Department of Industrial and Management System Engineering at West Virginia University.

\section{Purposes of the Study}

The purpose of this study is to investigate how people will react to different kind of sudden loadings, so as to develop better strategies to deal with them. WVU expects to enroll approximately 25 subjects; a total of approximately 25 subjects at all sites are expected to participate in this study.

\section{Description of Procedures}

Upon arrival the experiment will be described and you will be asked to sign this informed consent form. A brief ( 5 minutes) demonstration and exercise routine will be provided to let you familiar with the devices that you are going to use and the tasks that you are going to perform. You will then be fitted with 19 markers, which will be used to track the motion of 19 body segments. The markers will be placed over the following position: Right shoulder, Left shoulder, 7th cervical vertebra (C7), 12th thoracic vertebra (T12), 5th lumbar vertebra (L5), Right hip, Left hip, Right inner knee, Left inner knee, Right outer knee, Left outer knee, Right inner ankle, Left inner ankle, Right outer ankle, Left outer ankle, Right elbow, Left elbow, Right hand, and Left hand. You will then be fitted with a set of sensors designed to capture muscle activation levels (EMG), EMG sensor will be placed over the following muscles (right and left): lumbar erector spinae( $L 3)$, multifidus, external oblique and rectus abdominis. You will then sit on the platform of the dynamometer with their pelvis points and lower extremities secured and perform three trials of maximum flexion and extension tasks against a stationary resistance in a 20 degree trunk forward flexion posture. Each maximum voluntary contraction trial will be five seconds long and a minute's rest period will be provided between exertions in order to reduce the chance of fatigue and injury. You will then stand on the force plate, which is also the motion recording area and perform designated tasks. The design of experiment involves a total of three independent variables: three feet postures (close feet, open feet and separate feet), two levels of weight ( $7.5 \mathrm{lb}$ and $15 \mathrm{lb})$, and three load releasing position (symmetric, left 45 degree, right 45 degree). The

\begin{tabular}{lllll}
\hline Tracking \#: & H-24416 & Page 2 of 5 & & \\
Approved On: & $10 / 23 / 2012$ & & Date \\
Valid Through: & $10 / 22 / 2013$ & & \\
Last Amended: & N/A & &
\end{tabular}


Tracking \#: $\quad \mathrm{H}-24416$

combination of these three independent variables will create 14 conditions, and each condition will be repeated for 3 times which makes a total of 42 trials. The total 42 trials will be randomized, and 2 minutes of rest will be provided after every five trials to eliminate the possible muscle fatigue. Upon completion of all trials all markers and EMG sensors will be removed and you will be free to go.

\section{Risks and Discomforts}

There may be arms and low back muscle strain and fatigue while performing maximum exertions and other tasks. Therefore, you will be required to complete a warm up before these tasks and sufficient rest between trials.

\section{Alternatives}

You do not have to participate in this study.

The only alternative is not to participate in this study.

\section{Benefits}

You may not receive any direct benefit from this study. The knowledge gained from this study may eventually benefit others.

\section{Financial Considerations}

Subjects will not receive any compensation for participation in the study and will not incur any cost related to the study, It is very important for you to understand that neither the investigator nor WVU or it associated affiliates has the funds set aside to pay for the cost of lost work wages or any care or treatment that might be necessary because you get hurt or sick taking part in this study. Any injuries that may result from this study would not be eligible for Workers' Compensation as this is not a job related injury. Understand that any treatments necessary will be billed to the participant or to your personal health insurance, and you may wish to consult your insurance provider before participating in this study.

\section{Confidentiality}

Any information about you that is obtained as a result of your participation

\begin{tabular}{lllll}
\hline Tracking \#: & H-24416 & Page 3 of 5 & & \\
Approved On: & $10 / 23 / 2012$ & & Date \\
Valid Through: & $10 / 22 / 2013$ & & \\
Last Amended: & N/A & &
\end{tabular}


Tracking \#: $\quad \mathrm{H}-24416$

in this research will be kept as confidential legally possible. Your research records and test results, just like hospital records, may be subpoenaed by court order or may be inspected by federal regulatory authorities without your additional consent. In any publications that result from this research, neither your name nor any information from which you might be identified will be published without your consent.

\section{Voluntary Participation}

Participation in this study is voluntary. You are free to withdraw your consent to participate in this study at any time. Refusal to participate or withdrawal will not affect your future career, [or your employee status at West Virginia University or your class standing or grades, as appropriate] and will involve no penalty to you. In the event new information becomes available that may affect your willingness to participate in this study, this information will be given to you so that you can make an informed decision about whether or not to continue your participation. You have been given the opportunity to ask questions about the research, and you have received answers concerning areas you did not understand.

\begin{tabular}{lll}
\hline Tracking \#: & $\mathrm{H}-24416$ & Page 4 of 5 \\
Approved On: & $10 / 23 / 2012$ & \\
Valid Through: & $10 / 22 / 2013$ & \\
Last Amended: & N/A &
\end{tabular}


Upon signing this form, you will receive a copy.

I willingly consent to participate in this research.

$\begin{array}{lllll}{ } } & & \text { Date } & & \text { Time } \\ \text { Subjects Legal Representative } & & & \end{array}$

The participant has had the opportunity to have questions addressed. The participant willingly agrees to be in the study.

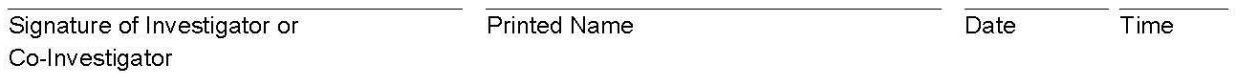

\begin{tabular}{lllll}
\hline Tracking \#: & H-24416 & Page 5 of 5 & & \\
Approved On: & $10 / 23 / 2012$ & & Date \\
Valid Through: & $10 / 22 / 2013$ & & \\
Last Amended: & N/A & &
\end{tabular}




\section{Only Minimal Risk Consent Information Form (without HIPAA)}

$\begin{array}{ll}\text { Principal Investigator } & \text { Xiaopeng Ning } \\ \text { Department } & \text { Industrial and Management Systems Engineering } \\ \text { Protocol Number } & 1309098696 \\ \text { Study Title } & \text { The effect of laterally slant ground on trunk biomechanical responses during sudden loading } \\ \text { Co-Investigator(s) } & \text { Jie Zhou } \\ \text { Sponsor (if any) } & \text { N/A }\end{array}$

\section{Contact Persons}

Click here to enter text.

In the event you experience any side effects or injury related to this research, you should contact Dr. Xiaopeng Ning at (304) 294-9474. (After hours contact: Dr. Xiaooeng Ning at (515) 520-1951). If you have any questions, concerns, or complaints about this research, you can contact Dr. Xiaopeng Ning or Mr. Jie Zhou at (304) 294-9474.

For information regarding your rights as a research subject, to discuss problems, concerns, or suggestions related to the research, to obtain information or offer input about the research, contact the Office of Research Integrity \& Compliance at (304) 293-7073.

In addition if you would like to discuss problems, concerns, have suggestions related to research, or would like to offer input about the research, contact the Office of Research Integrity and Compliance at 304-293-7073.

\section{Introduction}

You, have been asked to participate in this research study, which has been explained to you

by . This study is being conducted by Dr. Xiaopeng

Ning in the Department of Industrial and Management System Engineering at West Virginia University.

\section{Purpose(s) of the Study}

The purpose of this study is to investigate how people will react to sudden loading when standing on laterally slant ground, so as to develop better strategies to deal with them. We expect to enroll approximately 20 subjects; a total of approximately 20 subjects at all sites are expected to participate in this study.

\section{Description of Procedures}
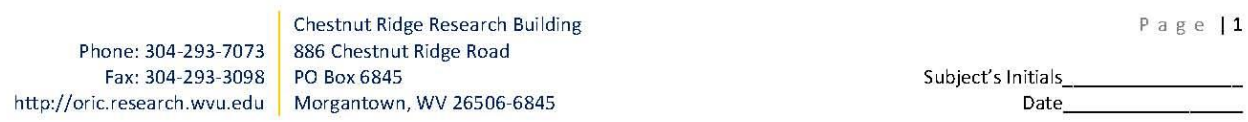
This study involves measurement of anthropometry, weight, height, trunk length, width and depth, performing 6 trials of trunk maximum voluntary contraction, performing 24 trials of sudden loading tasks, $7.5 \mathrm{lb}$ and $15 \mathrm{lb}$, when standing on laterally slanted ground surface, 0 degree, 15 degree and 30 degree. Motion sensors and muscle activation sensors will be attached on the skin of your trunk, and between each trial, ample rest will be provided to prevent muscle fatigue. After performing these trials and removing the sensors, you are free to leave. This procedure will take approximately 90 minutes for you to complete.

\section{Discomforts}

There may be a risk for low back muscle strain and fatigue while performing the trunk maximum exertions and other tasks. Therefore, you will be required to complete a warm up before these tasks and sufficient rest between trials.

\section{Alternatives}

You do not have to participate in this study.

\section{Benefits}

You may not receive any direct benefit from this study. The knowledge gained from this study may eventually benefit others.

\section{Financial Considerations}

You will not receive any compensation for participation in the study and will not incur any costs related to the study. It is very important for you to understand that neither the investigator nor WVU or it associated affiliates has the funds set aside to pay for the cost of lost work wages or any care or treatment that might be necessary because you get hurt or sick taking part in this study. Any injuries that may result from this study would not be eligible for Workers' Compensation as this is not a job related injury. Understand that any treatments necessary will be billed to the participant or to your personal health insurance, and you may wish to consult your insurance provider before participating in this study.

\section{Confidentiality}

Any information about you that is obtained as a result of your participation in this research will be kept as confidential as legally possible. Your research records and test results, just like hospital records, may be subpoenaed by court order or may be inspected by the study sponsor or federal regulatory authorities (including the FDA if applicable) without your additional consent

In any publications that result from this research, neither your name nor any information from which you might be identified will be published without your consent.

\section{Voluntary Participation}

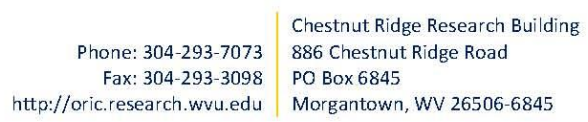

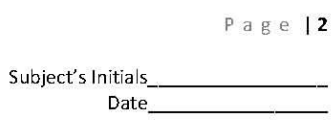




\section{W. WestVirginiaUniversity.}

Human Research Protocol Only Minimal Risk Consent Form

Without HIPAA

Participation in this study is voluntary. You are free to withdraw your consent to participate in this study at any time.

Refusal to participate or withdrawal will not affect [your class standing or grades, as appropriate] and will involve no penalty to you. Refusal to participate or withdrawal will not affect your future care, or your employee status at West Virginia University.

In the event new information becomes available that may affect your willingness to participate in this study, this information will be given to you so that you can make an informed decision about whether or not to continue your participation.

You have been given the opportunity to ask questions about the research, and you have received answers concerning areas you did not understand.

Upon signing this form, you will receive a copy.

I willingly consent to participate in this research.

\section{Signatures}

Signature of Subject

Printed Name

Date

Time

The participant has had the opportunity to have questions addressed. The participant willingly agrees to be in the study.

Signature of Investigator or Co-Investigator

Printed Name Date Time

Phone: 304-293-7073 886 Chestnut Ridge Road Fax: 304-293-3098 PO Box 6845 http://oric.research.wvu.edu Morgantown, WV 26506-6845 